\title{
Long-lived Excited State in a Solubilized Graphene Nanoribbon
}

Matthew C. Drummer ${ }^{1,2}$, Ravindra B. Weerasooriya ${ }^{1,2}$, Nikita Gupta ${ }^{1,2}$, Brian T. Phelan ${ }^{2,3}$, Andrew J. S. Valentine ${ }^{4}$, Amy A. Cordones ${ }^{5}$, Xiaosong $\mathrm{Li}^{4}$, Lin X. Chen ${ }^{2,3}$, and Ksenija D. Glusac $^{1,2^{*}}$

${ }^{1}$ Department of Chemistry, University of Illinois at Chicago, Chicago, Illinois 60607, United States

${ }^{2}$ Chemical Sciences and Engineering, Argonne National Laboratory, Illinois 60439, United States

${ }^{3}$ Department of Chemistry, Northwestern University, Evanston, Illinois 60208, United States

${ }^{4}$ Department of Chemistry, University of Washington, Seattle, Washington 98195-1700, United States

${ }^{5}$ Standard PULSE Institute, SLAC National Accelerator Laboratory, Menlo Park, California 94025, United States

\begin{abstract}
Graphene nanoribbons exhibit excellent light-absorbing properties, but often exhibit short excited-state lifetimes that prevent their applications in photocatalysis. Here, we report a longlived charge-transfer triplet excited state in a well solubilized, chlorinated graphene nanoribbon (Cl-GNR) with edges modified by bipyrimidine (bpm) moieties. The photophysical behavior of Cl-GNR was observed and characterized by steady-state UV-vis absorption and emission spectroscopy, transient absorption spectroscopy on the ps-ms timescale, and density functional theory (DFT) calculations. Both the Cl-GNR and its monomeric subunit, chlorinated graphene quantum dot (Cl-GQD), were synthesized using bottom-up techniques to produce the $\mathrm{H}$ - analogs of the compounds followed by edge-chlorination to achieve soluble products. The absorption spectra of Cl-GQD and Cl-GNR appear in the UV-vis range with lowest-energy peaks at 375 and $600 \mathrm{~nm}$, respectively. The excitons in Cl-GNR were found to exhibit charge-transfer character with the bpm edges serving as electron acceptors. DFT calculations indicate that the excitons are relatively localized, spreading over at most two monomeric units of the GNR. Transient absorption spectroscopy shows that singlet excited states of Cl-GQD and Cl-GNR undergo intersystem crossing with $\sim 300 \mathrm{ps}$ lifetime to form triplet state that lasts for $15.7 \mu \mathrm{s}(\mathbf{C l}-$ GQD) and $106 \mu$ s (Cl-GNR). These properties, combined with the ability of bpm sites to coordinate transition metals, make Cl-GNRs promising light-harvesting motifs for photocatalytic applications.
\end{abstract}

\section{Keywords}

graphene quantum dot, graphene nanoribbon, transient absorption spectroscopy, density functional theory, charge-transfer, triplet excited state, long-lived excited state

\section{Corresponding author's email}

glusac@uic.edu 


\section{Introduction}

Nanographenes, such as graphene quantum dots (GQDs) and nanoribbons (GNRs), are lightharvesting chromophores that exhibit desirable excitonic properties for application in photocatalysis. The relative size of GQDs and GNRs constrain their excitons to a size that is smaller than the essentially infinitely large exciton Bohr radius of bulk graphene.

Consequentially, excitons in these systems are quantum-confined, enabling size tunable energy gaps. ${ }^{1}$ Size-dependent tunability of the energy gap has been shown both theoretically ${ }^{2}$ and experimentally, ${ }^{3}$ demonstrating the versatility of light-harvesting in nanographenes. For example, triangular GQDs of varying size exhibit a progressive redshift of their ultranarrow absorption and emission bands with increasing size. ${ }^{3}$ Nanographene size/shape can also be utilized to tune their optical properties (and chemical stability) and is best described by the chemically intuitive Clar's sextet rule of polycyclic aromatic hydrocarbons which states that the more aromatic sextets (that is, the more benzene-like subunits) in the molecule, the larger the optical gap and higher the stability. ${ }^{4}$ Computational results confirm the sextet rule when comparing armchairand zigzag-edged nanographenes. Zigzag derivatives contain more nodes and less sextets than their armchair analogs, which results in the destabilization of their HOMO levels and a narrowing of their energy gaps. ${ }^{5}$ Chemical functionalization with electron-donating groups have also been used to systematically engineer the energy gap of GQDs for application in photocatalytic water splitting and $\mathrm{CO}_{2}$ reduction. ${ }^{6}$ These photocatalytic reactions have also been demonstrated for GQDs used in 0D/2D van der Waals heterojunctions with graphene. ${ }^{7}$ GQDs can also self-assemble into $\pi$-stacked structures that efficiently harvest light along the stack ${ }^{8-10}$. Furthermore, the photocatalytic properties of GQDs are enhanced upon coordination of a metal catalyst, such as cobaloximes for hydrogen evolution chemistry. ${ }^{11}$ Unlike GQDs, application of GNRs remains relatively unexplored, yet these compounds show great potential in photocatalysis since their absorption profiles cover much of the visible light region, making them efficient solar light absorbers. In addition to their excellent light harvesting, GNRs exhibit high charge carrier mobilities ${ }^{12}$ which are expected to enable efficient delivery of charge carriers to the oxidation and reduction catalytic sites for photocatalysis.

Recent developments in bottom-up nanocarbon synthesis methods have enabled the preparation of well-defined nanocarbons with tunable size, shape, and functional groups. ${ }^{13,14}$ These methods often start with the synthesis of soluble polyphenylene precursors using Diels-Alder addition/decarbonylation, ${ }^{15}$ Suzuki-Miyaura ${ }^{16}$, or Yamamoto ${ }^{17}$ coupling reactions. The aromatization of these soluble poly-phenylenes is achieved using oxidative cyclodehydrogenation to form insoluble nanographene products. ${ }^{18}$ More recently, one-shot annotative $\pi$-extension has been developed as a useful 'growth from template' method to selectively modify K, L and bay-regions of nanographenes using Diels-Alder or C-H activation chemistry. ${ }^{19,20}$ These synthetic tools have enabled numerous studies on the effect of nanographene size, shape and heteroatoms on the electronic and optical properties of welldefined carbon materials. ${ }^{21,22}$

Only a limited number of studies report the optical properties of GNRs. ${ }^{14,23-34}$ Using wet chemistry synthetic methods discussed above, several GNRs with 0.5-2 nm ribbon widths (where 
width is defined as the shortest edge-to-edge distance in the ribbon) were prepared and their optical bandgaps were reported to fall off with the increased ribbon width and to exhibit $1-2 \mathrm{eV}$ range. Such width-dependent optical properties are consistent with theoretically predicted quantum confinement effects in GNRs. Theoretical calculations also indicate that the width dependence on GNR bandgap depends on $\mathrm{C}$, the number of carbon atoms across the ribbon width. In GNRs with $\mathrm{C}=3 \mathrm{p}+1$ ( $\mathrm{p}$ is an integer), the fall-off of the optical bandgap with the ribbon width is more pronounced than that for GNRs with $\mathrm{C}=3 \mathrm{p}$ and $3 p+2 .{ }^{24}$ The optical gap was also found to be affected by the solubilizing alkyl chains owing to their steric effects induce the nanoribbon non-planarity and the reduction in the bandgap. ${ }^{25}$ The optical excitation of GNRs was found to be dominated by excitonic features, ${ }^{26}$ which is consistent with their quantum confinement and low dielectric screening, both of which prevent the exciton dissociation into free charge carriers. Free charge carriers were observed, using time-resolved $\mathrm{THz}$ spectroscopy, when the excess energy from the excitation pulse after excitation exceeded the exciton binding energy in GNRs $(\sim 700 \mathrm{meV}) .{ }^{27}$ These free carriers were found to exhibit excellent mobilities (in the $150-15,000 \mathrm{~cm}^{2} / \mathrm{V} \cdot \mathrm{s}$ range), but short lifetimes (few picoseconds). ${ }^{[15,29-31]}$ Excitons were also found to be highly mobile, as demonstrated by the presence of biexcitonic features in the pumpfluence dependent experiments. ${ }^{34}$ However, the exciton lifetimes were found to be relatively short-lived due to the excimer formation and the presence of dark states. ${ }^{32,33}$

As part of our ongoing interest in light-harvesting properties of nanographenes ${ }^{8,11,35}$, we investigate here the photophysical properties of hydrogenated and chlorinated GQDs and GNRs that contain bipyrimidine moieties in their edges (Scheme 1). Here, H-GQD and Cl-GQD can be thought of as the basic building blocks of H-GNR and Cl-GNR, respectively. In a previous electrochemical (non-photophysical) study, we showed that bipyrimidine units behave as redoxactive electron accepting moieties, undergoing efficient proton-coupled electron transfer processes. ${ }^{36}$ In this study, we show that GQD and GNR exhibit excited states with chargetransfer character, where bipyrimidine units serve as electron acceptors, an important property for efficient photoinduced charge separation required in photocatalytic applications. Furthermore, we find that the edge chlorination has a beneficial effect on the excited-state lifetimes of GNRs, due to the significant reduction in the non-radiative decay pathways associated with $\pi$-stacking between aromatic cores. These prolonged lifetimes also enable efficient intersystem crossing to produce long-lived (106 $\mu \mathrm{s})$ triplet states, a behavior that has never been observed previously in the graphene nanoribbon literature. These excellent photophysical properties, combined with the fact that GNRs absorb a broad range of visible frequencies and that the bipyrimidine sites can serve as coordinating sites for transition metalbased catalysts, makes these nanographenes excellent building blocks for future photocatalytic applications. 


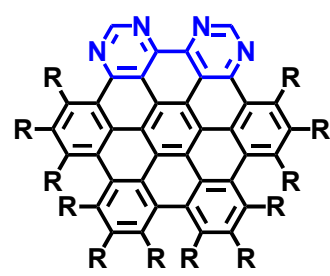

H-GQD: $\mathrm{R}=\mathrm{H}$ Cl-GQD: $\mathrm{R}=\mathrm{Cl}$

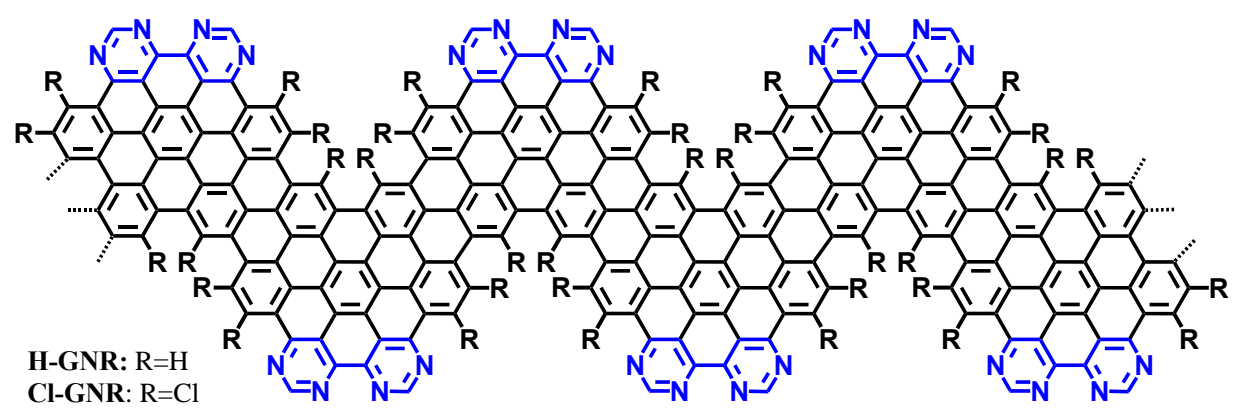

Scheme 1. Structures of hydrogenated (H-GQD and H-GNR) and chlorinated (Cl-GQD and ClGNR) nanographenes with bipyrimidine sites highlighted in blue..

\section{Results and Discussion}

\section{Synthesis and characterization.}

The synthesis of GQDs and GNRs is summarized in Scheme S1 and involves Diels-Alder chemistry, ${ }^{37}$ Yamamoto coupling polymerization ${ }^{38}$ and chlorination ${ }^{39}$ reactions that have been reported previously for similar substrates. The Diels-Alder reaction between cyclopentadienones $\mathbf{1}$ and acetylide $\mathbf{2}$, followed by a loss of $\mathrm{CO}$, resulted in the formation of polyphenylenes $\mathbf{3}$. The oxidative dehydrogenation of $\mathbf{3 H}$ using $\mathrm{FeCl}_{3}$ yielded $\mathbf{H - G Q D}$, while the subsequent chlorination using iodine-monochloride resulted in the formation of Cl-GQD. The NMR and MS analysis of Cl-GQD indicates that $\mathrm{C}-\mathrm{H}$ group between two $\mathrm{N}$-center of bipyrimidine unit are not chlorinated, while the remaining $\mathrm{C}-\mathrm{H}$ groups are mostly chlorinated ( $\sim 80 \%)$. The polymerization of 3-Br via Yamamoto coupling yields polymer-3, which is then oxidized to H-GNR using $\mathrm{FeCl}_{3}$. The MS characterization of polymer-3 ${ }^{36}$ indicates that ribbons up to $\sim 20 \mathrm{~nm}$ in length ( $163 \mathbf{3 H}$ units) are formed, while longer polymer-3 units, which may exist in the H-GNR sample, are not expected to get ionized and detected in the mass spectrometry. The ICl chlorination methods yield Cl-GNR, which have been characterized using XPS and NMR (Section S1, SI).

\section{Computational studies.}

While H-GNR was found to be insoluble in all solvents, it can be solubilized in aqueous solutions using surfactants, such as sodium dodecyl benzene sulfonate (SDBS). The UV-vis absorption spectra of H-GQD and H-GNR in aqueous SDBS solutions are shown in Figure 1a and consist of broad, structureless features that span a large portion of the UV and visible regions. H-GNR exhibits a lowest-energy absorption peak ( $\lambda=600 \mathrm{~nm}$ ) that is red-shifted relative to that of H-GQD $(\lambda=360 \mathrm{~nm})$, as expected based on the quantum confinement effect. To illustrate the effect of GNR length on the optical properties, we calculated electronic transition energies for GNR fragments with sizes in the monomer to pentamer range (Figure 1b, computational details are available in Section S4, SI). The calculated monomer spectrum consists of two absorption bands centered at 280 and $360 \mathrm{~nm}$. Geometry optimizations reveal substantial folding in the trimer and tetramer, such that the molecule is not entirely planar (Figure S12, SI). As the length of the model GNR increases from monomer to pentamer, both transitions undergo a red-shift and merge into one peak with absorption bands centered at 510 
$\mathrm{nm}$ for the pentamer. The red shift seems to saturate for GNR models longer than a tetramer, indicating that the exciton coherence length does not exceed beyond the length of $\sim 3.8 \mathrm{~nm}$. The lowest energy bright transitions are associated with the formation of charge-transfer (CT) excited state where the electron density shifts to the bipyrimidine edges, as illustrated in the difference density plots shown in Figure 1c. For trimer and tetramer GNR models, the inner bipyrimidine units accumulate the electron density, while the bipyrimidine units located on the periphery exhibit a slight depletion of electronic density while the majority of electron density depletion occurs within the aromatic core. The match between calculated $(360 \mathrm{~nm}, 3.44 \mathrm{eV})$ and experimental $(355 \mathrm{~nm}, 3.49 \mathrm{eV})$ absorption wavelengths for H-GQD is excellent, while the comparison of the H-GNR absorption band at $610 \mathrm{~nm}$ with the calculated data indicate that the H-GNR solution consists of GNRs with lengths that are longer than 5 monomer units.
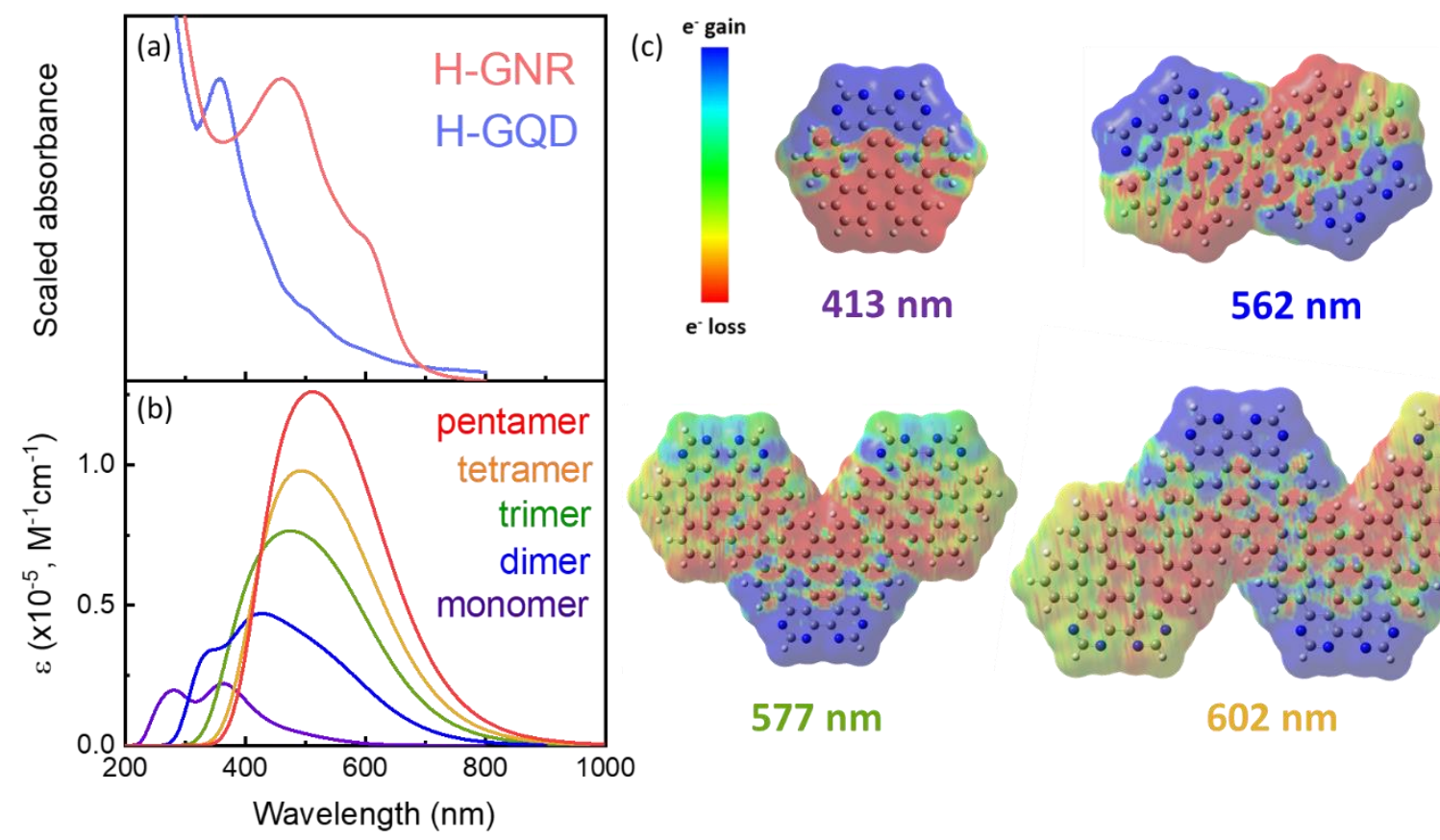

$562 \mathrm{~nm}$

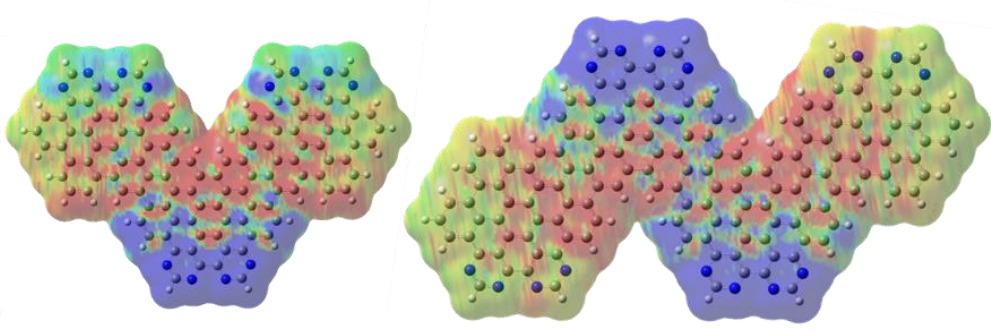

$577 \mathrm{~nm}$

$602 \mathrm{~nm}$

Figure 1. (a) Steady-state UV-vis absorption spectrum of H-GQD and H-GNR in aqueous solution with $1 \%$ wt. SDBS. (b) Time-dependent density functional theory (TD-DFT) calculated absorption spectra of H-GQD oligomers (monomer to pentamer). Calculations were performed at the B3LYP/6-31+G(d,p) level of theory with water solvation modeled by a polarizable continuum (iefpcm). (c) TD-DFT calculated difference density plots visualizing the ground-toexcited state electron density shift for the lowest-energy allowed transitions in monomer, dimer, trimer, and tetramer models.

The exciton size in GNRs was further investigated computationally using a one-particle transition density matrix approach developed previously. ${ }^{40}$ In this method, the exciton size $\mathrm{d}_{\mathrm{exc}}$ is defined as the root-mean-square separation between instantaneous hole and electron positions $\left(\vec{x}_{h}\right.$ and $\vec{x}_{e}$, respectively):

$$
d_{\text {exc }}=\sqrt{\left\langle\left|\vec{x}_{h}-\vec{x}_{e}\right|^{2}\right\rangle_{\text {exc }}}
$$


The value is calculated using transition density matrices for each excited state, under the assumption that the one-electron transition density matrix can be used as a representation of the exciton wavefunction. ${ }^{40}$ This approach was used to calculate $\mathrm{d}_{\mathrm{exc}}$ for $\mathrm{N}$-mers with sizes ranging from $N=1$ to 4 (Figure 2). The results show that the exciton size increases from $d_{\text {exc }}=5.3 \AA$ in the monomer to $7.1 \AA$ for tetramer, and the value seems to level off, with only a modest increase in size with $\mathrm{N}$-mer length. These results indicate that the exciton size in GNRs likely does not exceed beyond a dimer and are consistent with the previous calculations on a similar all-carbon GNR analog. ${ }^{41}$ At a first glance, these results appear contradictory to the continual red-shift observed in calculated UV-vis absorption spectra from monomer to pentamer. Such red-shift has often been interpreted in the literature as a measure of exciton size (the oligomer size at which the red-shift is no longer observed is used as an estimate of $\left.d_{\text {exc }}\right) .{ }^{42-46}$ However, our exciton size calculations in Figure 2 clearly show that the exciton size does not exceed beyond a dimer, even though the red-shift in Figure 1 is observed up to the pentamer model. Despite this, the TD-DFT spectra in Figure 1 will still capture shifts due to the electrostatic environment continuing to change as more material is added on either side, slowly converging according to the asymptotic tail of the coulomb force. The calculated natural transition orbitals (NTOs) also indicate that the exciton is not delocalizing past the dimer (Figure $2 b$ ). We obtained similar results in calculations of $\mathrm{d}_{\mathrm{exc}}$ in one-dimensional $\pi$-stacks of graphene flakes, ${ }^{8}$ indicating that the excitons do not delocalize significantly in these carbon-based chromophores, irrespective of the direction of their growth (along the $\pi$-stack in graphene flakes or via in-plane bonding in graphene ribbons). It may be noted that the particle-accepting NTO in the trimer appears to be localized over one monomer unit and therefore potentially smaller than the particle NTO of the dimer. However, the hole-leaving NTO is concomitantly larger in the dimer as well, allowing for the total holeparticle displacement to be smaller in the dimer than the trimer. To illustrate further the degree of hole-particle separation, transition density matrices in the tetramer were decomposed into contributions from natural atomic orbitals ${ }^{47}$; these may be found in the SI (S4). 
(a)

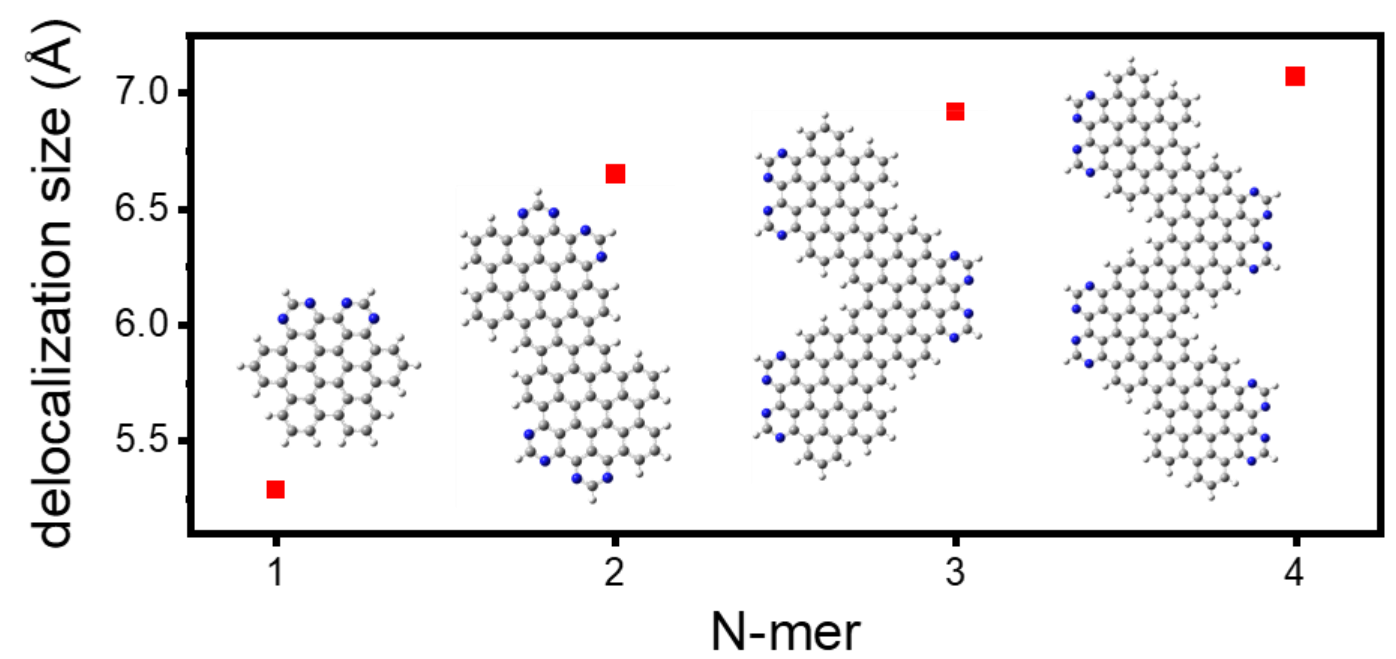

(b)
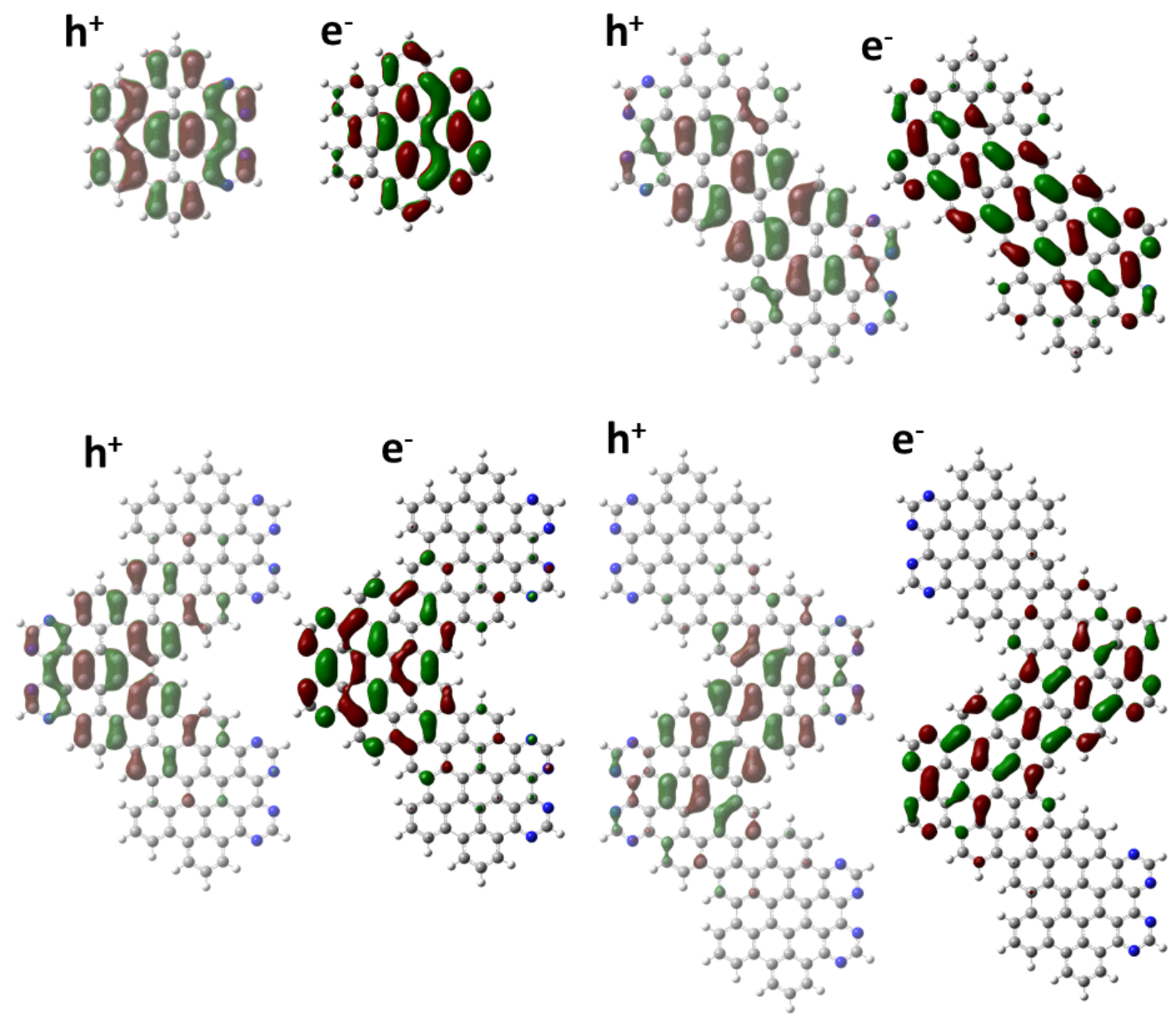

Figure 2. (a) Calculated exciton size as a function of the number of monomer units in the N-mer with structures shown in the inset. (b) Natural transition orbitals (NTO) of hole (transparent) and electron (opaque) of largest contribution are plotted over the structures for the lowest-energy bright state in each species. Calculation details are presented in the Section 4 of the SI. 


\section{Steady-state spectroscopy.}

Photoluminescence of H-GQD and H-GNR in aqueous surfactant solutions is very weak, as evident from the spectra presented in Figures $3 a$ and $b$. Since it is known that $\pi$-stacking causes a

significant increase in excited-state nonradiative decay rates in aromatic chromophores, ${ }^{8,48-50}$ we hypothesize that the use of aqueous surfactants results in solubilization of $\pi$-stacked aggregates of H-GQD and H-GNR, rather than fully dissolved isolated molecular species. Therefore, we attribute the steady-state spectroscopic signatures of H-GQD and H-GNR in Figure 2 to aggregates. To decrease $\pi$-stacking and improve solubilization of nanographenes, we utilized the chlorination methodology previously shown by Müllen and co-workers to be an effective strategy for functionalization of nanographene edges. ${ }^{39}$ The effect of chlorination on the nanographene structure is best observed when comparing the DFT-calculated structures of $\mathbf{H}$ GQD and CI-GQD (Figure 3c). While H-GQD is a planar molecule, the steric repulsion between chlorine atoms in Cl-GQD results in significant non-planarity. The chlorination of our model compounds significantly improved their solubility in organic solvents, such as toluene and trichlorobenzene, indicating reduced $\pi$-stacking interactions. Interestingly, the chlorination results in surprisingly small changes in the calculated UV-vis absorption spectra (Figure S13). Cl-GQD exhibits a modest bathochromic and hyperchromic shift relative to H-GQD, while the calculated difference density plots indicate that the lowest-energy transitions of both GQD derivatives are of the charge-transfer character (with bipyrimidine moieties acting as charge accepting sites). The experimental UV-vis absorption spectra are consistent with calculated predictions: H-terminated nanographenes H-GQD and H-GNR exhibit absorption spectra with absorption maxima located at similar energies as the spectra of Cl-GQD and Cl-GNR, respectively (Figures $3 \mathrm{a}$ and $3 \mathrm{~b}$ ), with slightly red-shifted absorption maxima. However, the effect of chlorination is more drastic when the photoluminescence spectra are compared. While H-GQD and H-GNR exhibit almost negligible emission, relatively strong emission peaks are observed for Cl-GQD and Cl-GNR in the visible range (quantum yields are 1\% and 15\%, respectively. See Section $\mathrm{S} 2$ for more detail). The emission is assigned to the fluorescence from singlet excited states of Cl-GQD and Cl-GNR, based on their insensitivity to oxygen (Figure S9). 


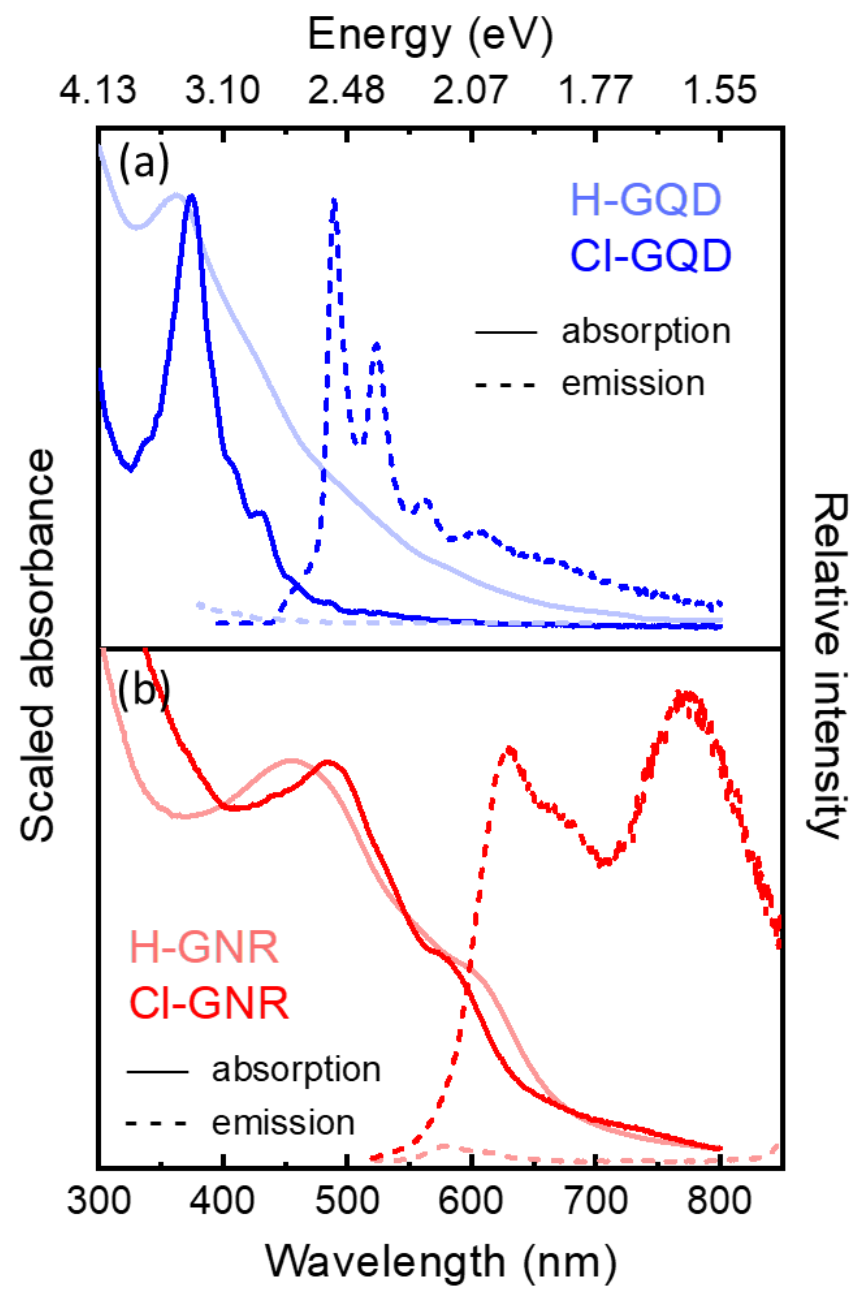

(c)
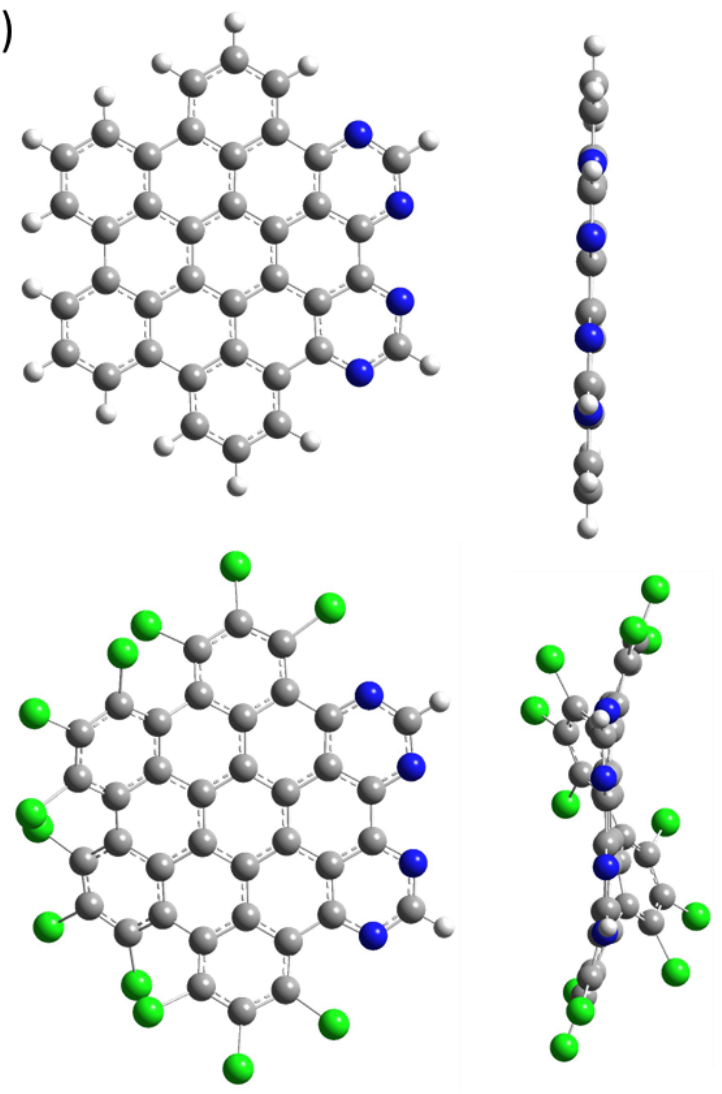

Figure 3. (a) UV-vis absorption (solid lines) and emission (dashed lines) spectra of H-GQD in aqueous $1 \%$ wt. SDBS (light blue) and Cl-GQD in toluene (dark blue). Emission spectra were collected upon excitation at $\lambda_{\mathrm{exc}}=360 \mathrm{~nm}$ (H-GQD) and $375 \mathrm{~nm}$ (Cl-GQD). (b) UV-vis absorption (solid lines) and emission (dashed lines) spectra of H-GNR in aqueous $1 \%$ wt. SDBS (light red) and Cl-GNR in trichlorobenzene (dark red). Emission spectra were collected upon excitation at $\lambda_{\mathrm{exc}}=500 \mathrm{~nm}$. (c) Structures of H-GQD (top) and Cl-GQD (bottom) optimized at the B3LYP/6-31+G(d,p) level of theory.

Steady-state excitation/emission spectroscopy provides some evidence for sample aggregation, even for nanographenes with chlorinated edges. Figure 4 shows 2D emission/excitation maps for Cl-GQD and Cl-GNR, along with excitation and emission spectra at selected wavelengths. In the case of Cl-GQD, this effect is best observed in normalized emission spectra in Figure 4b, which show that the excitation at $360 \mathrm{~nm}$ gives rise to an emission spectrum dominated by the sharp feature maximum at $490 \mathrm{~nm}$ and a weak contribution from the broad features at $625 \mathrm{~nm}$. As the excitation wavelength move to lower energy, the relative intensity of the $625 \mathrm{~nm}$ feature increases. Quite similar emission profiles consisting of intense and skinny bands in the higher energy range and broad emission features at lower energies were observed by us and others 
concentration-dependent emission spectra of analogous GQDs. ${ }^{8,51-53}$ Based on this similarity, we assign the $500 \mathrm{~nm}$ band to the emission from monomeric Cl-GQD, while the emission at $625 \mathrm{~nm}$ is assigned to the $\pi$-stacked aggregate of Cl-GQD. The absorption profiles of monomeric and aggregated Cl-GQD can be derived from the excitation spectra collected at monomer emission $(490 \mathrm{~nm})$ and aggregate emission $(625 \mathrm{~nm})$ wavelengths. The excitation spectrum assigned to the monomeric species exhibits an absorption maximum at $375 \mathrm{~nm}$. The excitation spectrum assigned to the aggregate exhibits a much broader, featureless signature that ranges from 300$475 \mathrm{~nm}$. Again, these excitation profiles are similar to the electronic transitions observed in the variable-concentration absorption spectra reported previously for analogous GQDs, ${ }^{8,51-53}$ further confirming our assignment.

Similar sample heterogeneity was observed in emission excitation maps of Cl-GNR. Figure $4 \mathrm{e}$ shows that the relative intensity of emission peaks at 650 and $775 \mathrm{~nm}$ changes with the excitation wavelength and we accordingly assign the $650 \mathrm{~nm}$ emission to the monomeric nanoribbons and the $775 \mathrm{~nm}$ emission to the aggregated Cl-GNR. The excitation spectra collected at the wavelengths for monomer and aggregate emission (Figure 4f) indicate that both species contain a main absorption feature at $\sim 500 \mathrm{~nm}$ and a shoulder at $600-700 \mathrm{~nm}$. The aggregate absorption features are slightly red-shifted and significantly broader, which is commonly observed in $\pi$ stacked aggregates. The kinetic profiles for the emission at the two bands (collected at 630 and $770 \mathrm{~nm}$, Figure S9, SI) also differ, confirming the sample heterogeneity. Both emission kinetics could be fit to a model involving one rise component with a lifetime of $0.5 \mathrm{~ns}$ and two decay components with lifetimes of 0.6 and $2 \mathrm{~ns}$. However, the relative amplitudes associated with these components were different at 630 and $770 \mathrm{~nm}$ with the longer-lived $2 \mathrm{~ns}$ component exhibiting much stronger amplitude at $770 \mathrm{~nm}$. Since CI-GNR samples consist of ribbons with different lengths, the heterogeneity observed in Cl-GNR emission/excitation maps may also be associated with the emission from non-aggregated ribbons of different lengths. However, given the similar heterogeneous emission observed in Cl-GQD and Cl-GNR, it is more likely that the observed behavior originates from aggregation effects. This is also supported by the calculated absorption spectra for different ribbon oligomers (Figure 1b), which indicate that any ribbon longer than four monomer units is expected to exhibit very similar photophysical properties. 

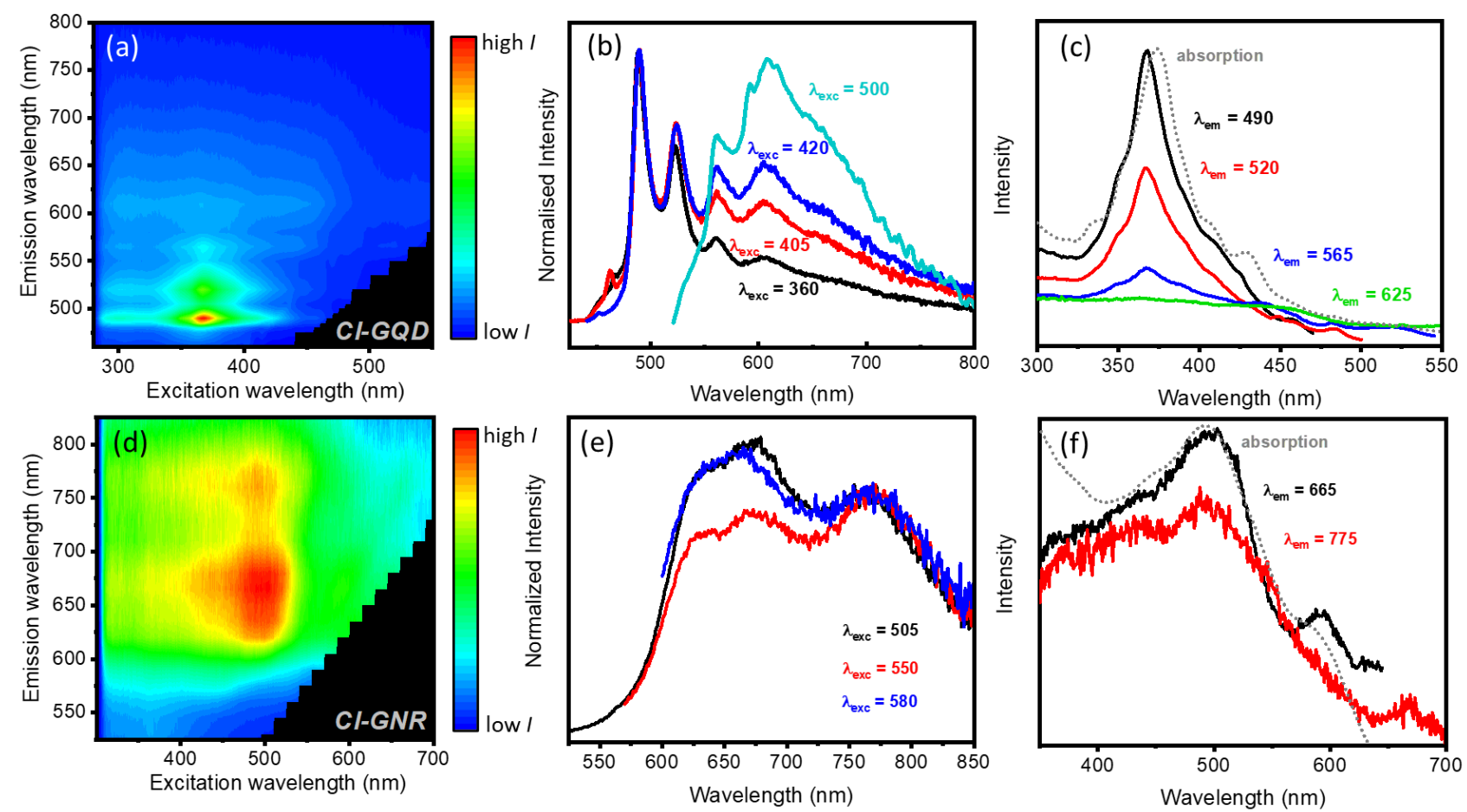

Figure 4. 2D emission/excitation maps of (a) Cl-GQD in toluene and (d) Cl-GNR in trichlorobenzene. Emission spectra at various excitation wavelengths for (b) Cl-GQD and (e) ClGNR. Excitation spectra at various emission wavelengths for (c) Cl-GQD and (f) Cl-GNR with absorption spectra overlaid.

Femtosecond transient absorption spectroscopy was employed to study the time-resolved photophysics of our $\mathrm{H}$ - and $\mathrm{Cl}$ - compounds (Figure 5). Transient features of all four samples at early probing times consist of ground-state bleach signals at $\sim 400 \mathrm{~nm}$ (for GQD samples) and $\sim 500 / 600 \mathrm{~nm}$ (for GNRs) with broad and featureless excited-state absorption bands in the redder part of the visible range. We assign the positive features to the excited-state absorption of singlet $\mathrm{S}_{1}$ states of GQDs and GNRs. Despite similar spectra features at early times, the temporal evolution of $\mathrm{H}$-compounds is drastically different from $\mathrm{Cl}$-compounds. While most of the transient signal of $\mathrm{H}$-compounds decays back to ground state within a few hundreds of picoseconds, the decays of $\mathrm{S}_{1}$ states of $\mathrm{Cl}$-compounds are longer and associated with a growth of new long-lived transient species with absorption at $\sim 550 \mathrm{~nm}$ (for Cl-GQD) and $\sim 700 \mathrm{~nm}$ (for ClGNR). These results are consistent with the increased fluorescence quantum yields observed for chlorinated compounds in Figure 3 and further confirms that the chlorination of nanographene edges represents an efficient method to prevent excited-state lifetime shortening associated with the $\pi$-stacking of aromatic units. ${ }^{54}$ 

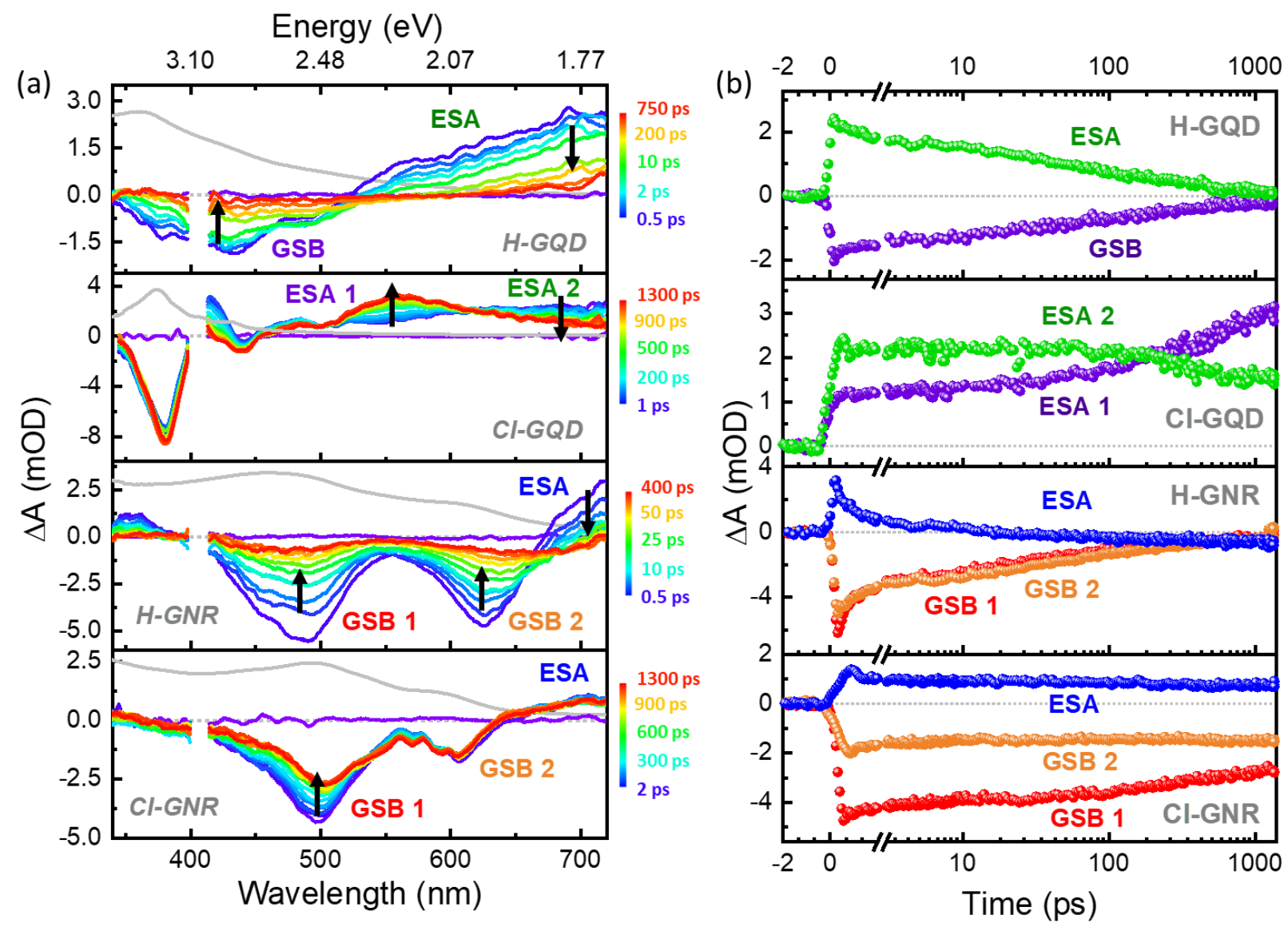

Figure 5. Transient absorption (a) spectra and (b) kinetics plots for H-GQD, CI-GQD, H-GNR, and Cl-GNR. Both H- compounds were measured in aqueous solution with $1 \%$ wt SDBS, ClGQD in toluene, and Cl-GNR in trichlorobenzene. All samples were excited at $400 \mathrm{~nm}$.

The formation of a long-lived transient with absorption at $\sim 550 \mathrm{~nm}$ in Cl-GQD occurs with a lifetime of $350 \pm 10 \mathrm{ps}$, as determined using the target analysis of the experimental data (Figure $6 a)$. The kinetic model used in target analysis was a simple $1 \rightarrow 2 \rightarrow 3$ sequential model and the resulting component spectra match the evolving features at 550 and $700 \mathrm{~nm}$ in the experimental data (Figure 6b). The temporal evolution of the long-lived transient was probed using nanosecond transient absorption spectroscopy (Figures $6 \mathrm{c}$ and d). The transient spectrum obtained at early times of the nanosecond instrument matches very well with the spectrum obtained at the end of the femtosecond instrument (probed 1.3 ns after the excitation pulse), confirming that the same intermediate is monitored across the two instrumental setups. The nanosecond instrument reveals that the long-lived intermediate decays with a lifetime of $15.7 \mu \mathrm{s}$ in the $\mathrm{N}_{2}$-purged sample, while it is significantly shorter in the presence of oxygen $(0.76 \mu \mathrm{s}$, Figure 6d). The long-lived nature of this transient, coupled with its sensitivity to molecular oxygen, indicates that this transient is likely the triplet excited $\mathrm{T}_{1}$ state of Cl-GQD. The Jablonski diagram in Figure 6c summarizes the observed photophysical behavior of Cl-GQD. 

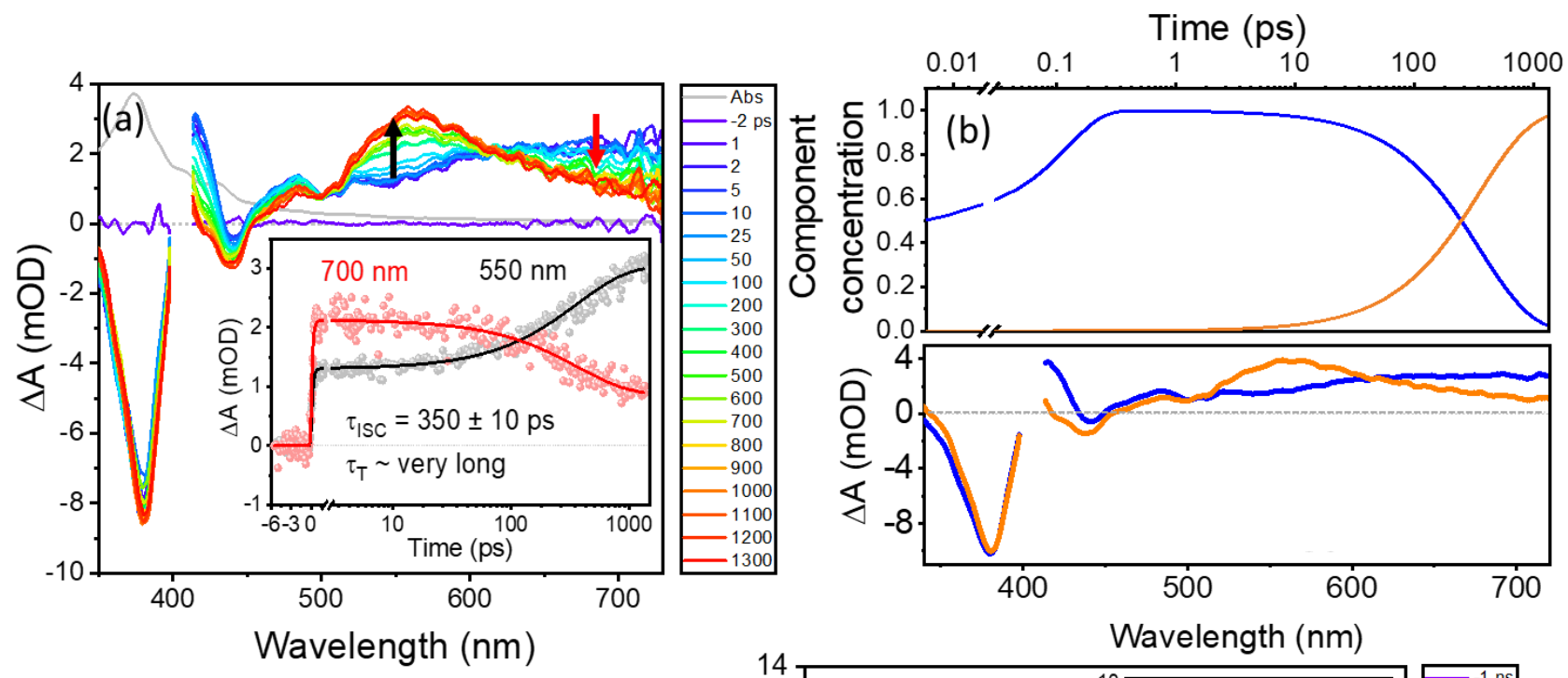

(c)
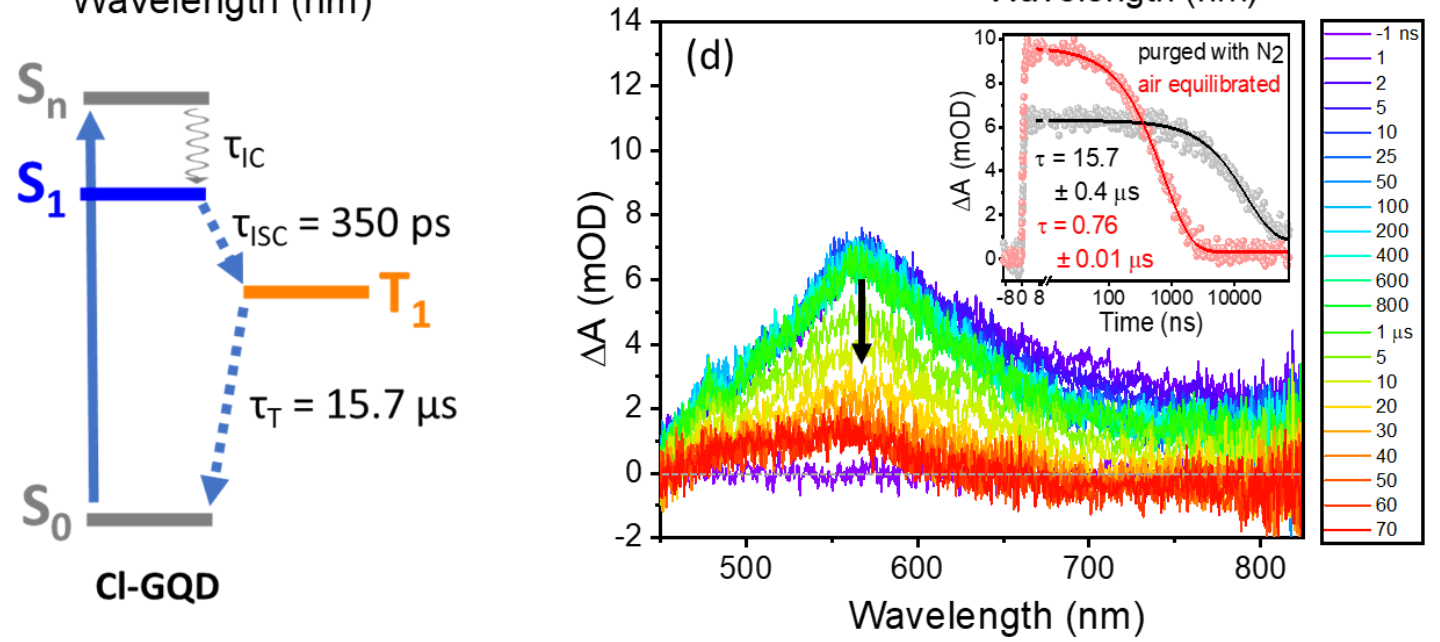

Figure 6. (a) Femtosecond transient absorption (TA) spectra of Cl-GQD in toluene probed at different times after the $\lambda_{\text {exc }}=400 \mathrm{~nm}$ excitation pulse; inset shows kinetic traces of the femtosecond data at bleach wavelength $(550 \mathrm{~nm})$ and excited state absorption wavelength (700 $\mathrm{nm}$ ) (b) Component concentrations as a function of time (top) and component spectra (bottom) obtained via target analysis using a sequential $1 \rightarrow 2 \rightarrow 3$ kinetic model. (c) Jablonski diagram describing the kinetic model used in the global fitting analysis. (d) Nanosecond transient absorption spectra of Cl-GQD in toluene obtained at different time delays after the $\lambda_{\text {exc }}=410 \mathrm{~nm}$ excitation pulse; inset shows fitting of kinetic trace at $550 \mathrm{~nm}$ with (red) and without (black) oxygen present.

The observation of efficient intersystem-crossing in CI-GQD is somewhat unusual considering that the molecule consists of light elements that cannot induce significant spin-orbit coupling via the common heavy-atom effect. ${ }^{55}$ In general, the rate of intersystem crossing can be expressed within the Fermi golden rule approximation as: ${ }^{56}$

$$
k_{I S C}^{F C}=2 \pi \sum_{\alpha}\left|\left\langle\Psi_{S_{a}}\left|\widehat{\mathcal{H}}_{S O}\right| \Psi_{T_{b}}^{\alpha}\right\rangle\right|_{q_{0}}^{2} \rho\left(E_{a j}\right)
$$


Where $\Psi_{S_{a}}$ and $\Psi_{T_{b}}^{\alpha}$ are the initially populated singlet state and ISC-formed triplet state, respectively. $\widehat{\mathcal{H}}_{S O}$ is the spin-orbit operator and $\rho\left(E_{a j}\right)$ is the vibrational density of states at the energy of the initial state, $\Psi_{S_{a}}$. The subscript $q_{0}$ denotes that the integral is solved for when $\Psi_{S_{a}}$ and $\Psi_{T_{b}}^{\alpha}$ have the same equilibrium geometry, i.e. the crossing point of their potential energy surfaces. Based on this expression, the rate of intersystem crossing can be enhanced by one of the two parameters: (i) by increasing the spin-orbit coupling matrix element or (ii) by increasing the density of states with small $\mathrm{S}-\mathrm{T}$ splitting $\left(\Delta \mathrm{E}=\mathrm{E}_{\mathrm{S}}-\mathrm{E}_{\mathrm{T}}\right)$. The spin-orbit coupling matrix was shown both experimentally and computationally to increase in non-planar polyaromatic hydrocarbons, such as helicenes. ${ }^{57,58}$ Computational analysis indicates that this increase is associated with the enhancements of the spin-orbit coupling matrix element, due to lower symmetry of nonplanar molecules: planar molecules contain molecular plane of symmetry, so only the out-of-plane component of their angular moment operator contributes to antisymmetric $\pi, \pi^{*}$ states. In contrast, nonplanar molecules lack the plane of symmetry, enabling nonvanishing matrix element contributions from all three components of the angular moment operator. ${ }^{58}$ Given that chlorination of GQDs results in a loss of planarity in CI-GQD (Figure 3C), we considered the possibility that the observed efficiency of intersystem crossing arises due to the nonplanarity of Cl-GQD. This hypothesis was tested by comparing the photophysical behavior of H-GQD and CI-GQD in organic solvents. The solubility of H-GQD (unlike H-GNR) in organic solvents (such as $o$-dichlorobenzene) was sufficient to enable a direct comparison of transient absorption features of the planar H-GQD (Figure S10) and nonplanar Cl-GQD (Figure 6). Interestingly, HGQD in organic solution (Figure S10) exhibits similar photophysical behavior as Cl-GQD, with an intersystem crossing time constant of $350 \pm 13$ ps (the value is $\sim 200$ ps for H-GQD). The longlived character observed of H-GQD in organic solvent allows us to conclude that the compound had better solubility than in aqueous solution. Based on this finding, we conclude that the nonplanarity of GQDs is not responsible for the observed triplet state formation. Thus, we assign the observed triplet formation yields to the second term in the equation 2: an increased density of states with small S-T splitting (in the weak coupling regime, the "energy gap law" dictates an exponential decay of the transition probability as $\Delta \mathrm{E}$ increases. ${ }^{56}$ The increased delocalization in graphene quantum dots is known to result in improved intersystem-crossing rates, which was attributed to a decrease in the S-T energy gaps. ${ }^{59}$ We hypothesize that a similar effect explains the efficient triplet formation in CI-GQD.

Like Cl-GQD, the Cl-GNR exhibits long-lived photo-generated intermediates (Figure 7). Transient absorption spectra of Cl-GNR consist of two ground-state bleach peaks at 500 and 600 $\mathrm{nm}$, as well as a broad excited-state absorption feature at $\sim 700 \mathrm{~nm}$. The 500 and $600 \mathrm{~nm}$ bleach bands undergo red and blue-shifts, respectively, during the first nanosecond after the excitation pulse (Figure 7A), while the measurements in the longer time-window show a decay of the signal on the microsecond timescale (Figure 7D). Global fitting of these transient features using a sequential $1 \rightarrow 2 \rightarrow 3 \rightarrow 4$ kinetic model provided satisfactory reproduction of the kinetic behavior (solid lines in the insets of Figures 7A and D) with the following lifetimes: $0.6 \mathrm{ps}, 6 \mathrm{ps}$, $300 \mathrm{ps}$ and $106 \mu \mathrm{s}$. The $0.6 \mathrm{ps}, 300 \mathrm{ps}$ and $106 \mu$ s lifetimes are like those observed in CI-GQD and are accordingly assigned to the $S_{n} \rightarrow S_{1}$ internal conversion $(0.6 \mathrm{ps}), S_{1} \rightarrow T_{1}$ intersystem crossing (300 ps) and $\mathrm{T}_{1} \rightarrow \mathrm{S}_{0}$ deactivation $(106 \mu \mathrm{s})$, as summarized by the Jablonski diagram in 
Figure 7c with component concentrations and spectra shown in Figure 7b. The assignment of the long-lived $106 \mu$ s component to the $\mathrm{T}_{1}$ state of Cl-GNR is consistent with the spin-forbidden nature of the deactivation back to the singlet ground state and is supported by its sensitivity to molecular oxygen (Figure $7 d$ ).

The 6 ps component is unique to CI-GNR samples and has not been observed in Cl-GQDs. We considered the possibility that this kinetic component is associated with the recombination of free charge carriers formed upon photoexcitation. Previous reports indicate that the exciton binding energies in nanographenes range from 0.1 to $0.6 \mathrm{eV}$ for GQDs ${ }^{6,7,60}$ and 0.5 to $2.5 \mathrm{eV}$ for GNRs. ${ }^{61}$ Furthermore, time-resolved microwave experiments show that the free carriers are formed in nanographenes when excitation pulse energies exceed the exciton binding energy and that these carriers recombine on picosecond timescales. ${ }^{27}$ To explore whether our 6 ps decay is associated with recombination of free charge carriers, we performed TA experiments using different excitation pulse energies (Figure S11, SI). If this component arises due to charge carrier recombination, it will disappear from the spectra obtained at low excitation energies, because the excitation pulse is not energetic enough to dissociate excitons into free carriers. Our data clearly show that the 6 ps component does not disappear from the transient decays, even when a very low energy excitation pulse $\left(\lambda_{\text {exc }}=600 \mathrm{~nm}\right)$ is used, indicating that this process is not associated with free charge-carriers. A more plausible origin of the 6 ps component is the excited-state decay of aggregated Cl-GNR. Based on the observed aggregation of nanoribbons in 2D excitation/emission spectra of Cl-GNR, it is quite likely that the observed transient data contain contributions from both isolated and aggregated ribbons. Since the aggregated ribbons are expected to exhibit short excited-state lifetimes, $, 81,52,62$ the 6 ps component is assigned to the excited states of aggregated Cl-GNRs (Figure 7c). This assignment is consistent with our observations of H-GNR which exhibits rapid decay on the single to tens of picoseconds timescale. 

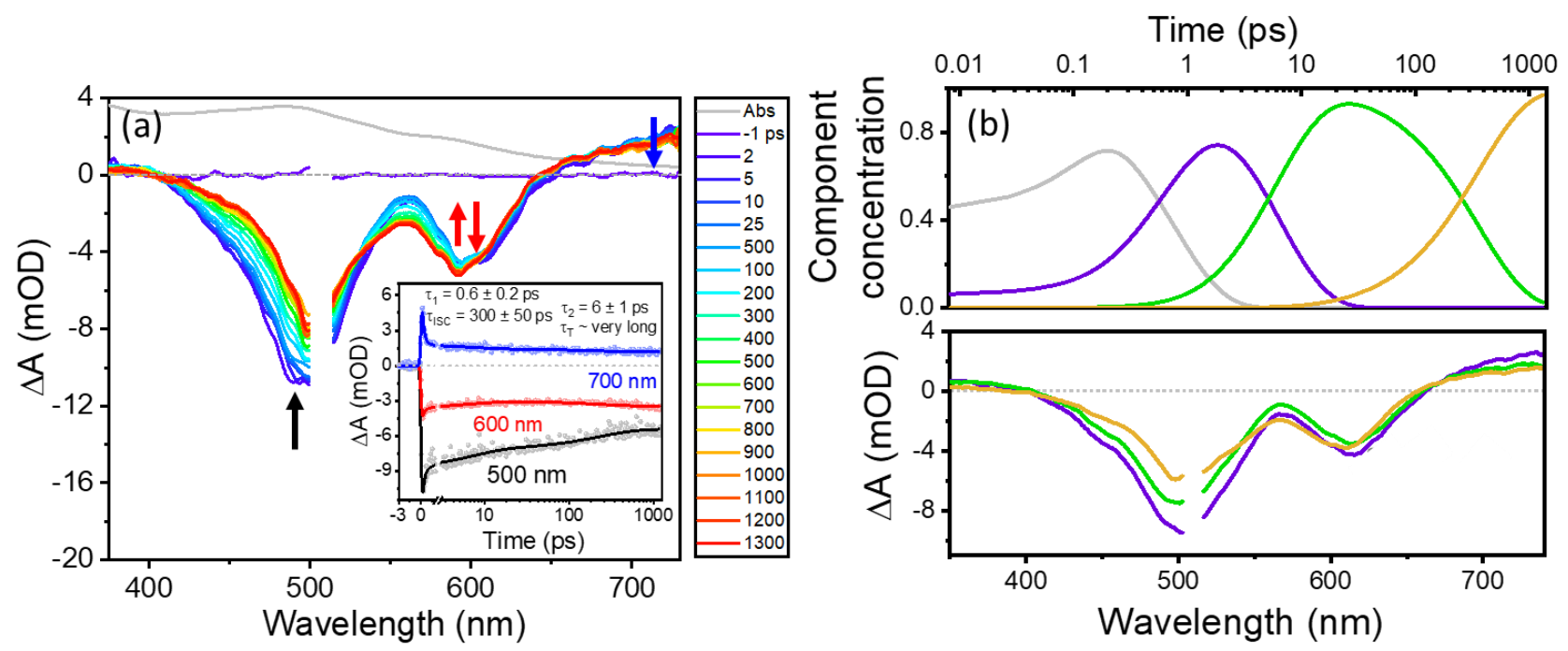

(c)

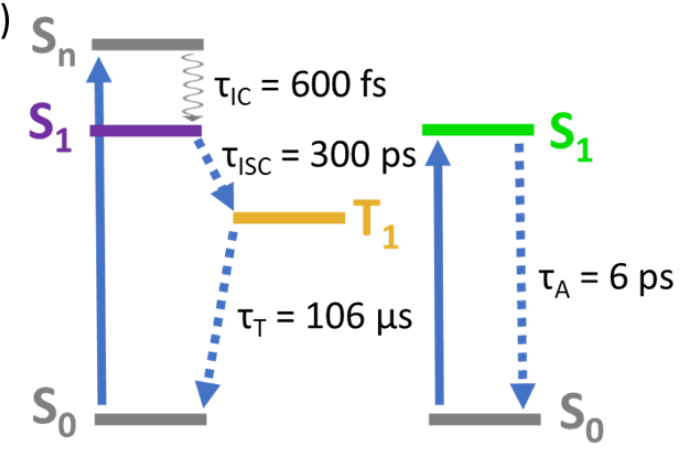

isolated Cl-GNR

aggregated Cl-GNR

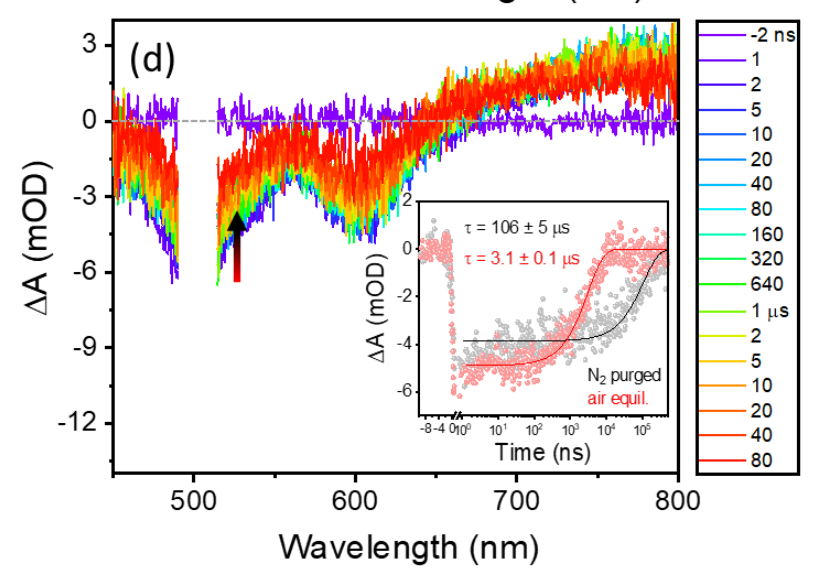

Figure 7. (a) Femtosecond transient absorption (TA) spectra of CI-GNR in trichlorobenzene probed at different times after the $\lambda_{\text {exc }}=500 \mathrm{~nm}$ excitation pulse; inset shows kinetic traces of the femtosecond data at bleach wavelengths $(500$ and $600 \mathrm{~nm})$ and excited state absorption wavelength $(700 \mathrm{~nm})$. Dashes represent experimental data, while the solid lines represent the fits obtained using target analysis. (b) Temporal (top) and spectral (bottom) profiles of components obtained via target analysis using a sequential $1 \rightarrow 2 \rightarrow 3 \rightarrow 4$ kinetic model. (c) Jablonski diagram of the proposed kinetic model. The model used in global fitting analysis was a simple sequential model. (d) Nanosecond TA spectra of Cl-GNR in trichlorobenzene obtained at different time delays after the $\lambda_{\text {exc }}=500 \mathrm{~nm}$ excitation pulse. Inset shows fitting of kinetic trace at $500 \mathrm{~nm}$ with (red) and without (black) oxygen present. Dashes represent experimental data, while the solid lines represent the fits obtained using target analysis.

The observed formation of long-lived triplet states in CI-GNR is quite unique. All previous studies of graphene nanoribbon photophysics report relatively short-lived singlet excited states with lifetimes on the order of $\sim 100$ ps. $^{27,34}$ The observed $106 \mu$ s lifetime is excellent for potential photocatalysis applications where long-lived excited states are required to provide sufficient time for useful chemical reactions to take place. The charge-transfer character of H-GNR excited states (Figure 1c) is a direct consequence of electron accepting properties of bipyrimidine 
moieties ${ }^{36}$ and enables spatial separation of electrons and holes within the exciton, which is useful for photoinduced electron transfer processes that initiate most photocatalytic reactions. We expect similar charge-transfer states in Cl-GNR given the similarities between H-GQD and Cl-GQD difference density plots (Figure S14, SI). In addition to exhibiting long-lived triplet excited states, Cl-GNR absorb very well in the visible range (Figure 3), making them excellent light-harvesting chromophores. Based on the computational results presented here, we speculate that the triplet states in Cl-GNR are charge-transfer in character and, therefore, well-suited for application in photocatalysis. Furthermore, the observed optical bandgap in Cl-GNR $(\sim 2 \mathrm{eV})$ is ideal for solar fuel applications, such as photochemical water splitting or $\mathrm{CO}_{2}$ reduction, as it provides enough energy for required reaction thermodynamics (for example, $1.23 \mathrm{eV}$ for solar water splitting) and for associated kinetic overpotentials (usually few hundred meVs). Finally, the bipyrimidine edges on Cl-GNR can serve as coordination sites for molecular transition metal catalysts for future applications in photocatalysis, as many of reported catalysts utilize polypyridyl ligands. ${ }^{63-68}$ Given all these arguments, the photophysical findings presented here illustrate the potential of Cl-GNR for applications in solar energy conversion systems. ${ }^{69}$

\section{Conclusion}

In conclusion, we report a detailed investigation of electronic properties of two nanographenes, GQD and GNR. We find that these model compounds can be solubilized into aqueous solutions using surfactants and into organic solvents using edge chlorination. The nanographenes in aqueous media were found to aggregate, causing significant shortening of their excited-state lifetimes. The chlorinated samples, despite a certain degree of aggregation, were found to exhibit long-lived charge-transfer excited states. Our computational studies indicate that the excitonic features in GNRs are delocalized over at most monomeric units and that they exhibit chargetransfer character, with bipyrimidine moieties acting as electron-accepting units. The absorption spectra of GNRs span a large portion of the visible, up to $700 \mathrm{~nm}$, making them promising lightharvesting motifs. Time-resolved experiments reveal that Cl-GNR and Cl-GNR undergo efficient ISC, probably facilitated by large density of electronic states, to form long-lived triplet excited states (15.7 and $106 \mu \mathrm{s}$, respectively). We anticipate that this study will inspire future work involving coordination of metal catalysts to the bipyrimidine sites of Cl-GNRs and further investigations into the fundamental photophysics of such GNR/catalyst assemblies and their applications in photocatalysis.

\section{Supporting Information}

Details on general methods, synthesis, characterization, steady-state spectroscopy, transient absorption spectroscopy, and computational methods. This material is available free of charge via the Internet at https://pubs.acs.org.

\section{Acknowledgments}

This work is supported by the U.S. Department of Energy (DOE), Office of Science, Office of Basic Energy Science, Division of Chemical Sciences, Geosciences and Biosciences, through Argonne National Laboratory under Contract No. DE-AC02-06CH11357. K.D.G. also acknowledges the support provided by National Science Foundation (Grant Number 1954298). 
Special thanks to Jonathan Gesiorski for the brilliant suggestion of chlorination to achieve solubility of the nanographenes in this study.

\section{References}

(1) Li, L.; Yan, X. Colloidal Graphene Quantum Dots. J. Phys. Chem. Lett. 2010, 1 (17), 2572-2576. https://doi.org/10.1021/jz100862f.

(2) Zhang, Z. Z.; Chang, K.; Peeters, F. M. Tuning of Energy Levels and Optical Properties of Graphene Quantum Dots. Phys. Rev. B 2008, 77 (23), 235411. https://doi.org/10.1103/PhysRevB.77.235411.

(3) Yuan, F.; Yuan, T.; Sui, L.; Wang, Z.; Xi, Z.; Li, Y.; Li, X.; Fan, L.; Tan, Z.; Chen, A.; Jin, M.; Yang, S. Engineering Triangular Carbon Quantum Dots with Unprecedented Narrow Bandwidth Emission for Multicolored LEDs. Nat. Commun. 2018, 9 (1), 2249. https://doi.org/10.1038/s41467-01804635-5.

(4) Clar, E. The Aromatic Sextet. In Mobile Source Emissions Including Policyclic Organic Species; Rondia, D., Cooke, M., Haroz, R. K., Eds.; Springer Netherlands: Dordrecht, 1983; pp 49-58. https://doi.org/10.1007/978-94-009-7197-4_4.

(5) Yamijala, S. S. R. K. C.; Mukhopadhyay, M.; Pati, S. K. Linear and Nonlinear Optical Properties of Graphene Quantum Dots: A Computational Study. J. Phys. Chem. C 2015, 119 (21), 12079-12087. https://doi.org/10.1021/acs.jpcc.5b03531.

(6) Yan, Y.; Chen, J.; Li, N.; Tian, J.; Li, K.; Jiang, J.; Liu, J.; Tian, Q.; Chen, P. Systematic Bandgap Engineering of Graphene Quantum Dots and Applications for Photocatalytic Water Splitting and $\mathrm{CO}_{2}$ Reduction. ACS Nano 2018, 12 (4), 3523-3532. https://doi.org/10.1021/acsnano.8b00498.

(7) Yan, Y.; Zhai, D.; Liu, Y.; Gong, J.; Chen, J.; Zan, P.; Zeng, Z.; Li, S.; Huang, W.; Chen, P. Van Der Waals Heterojunction between a Bottom-Up Grown Doped Graphene Quantum Dot and Graphene for Photoelectrochemical Water Splitting. ACS Nano 2020, 14 (1), 1185-1195. https://doi.org/10.1021/acsnano.9b09554.

(8) Singh, V.; Zoric, M. R.; Hargenrader, G. N.; Valentine, A. J. S.; Zivojinovic, O.; Milic, D. R.; Li, X.; Glusac, K. D. Exciton Coherence Length and Dynamics in Graphene Quantum Dot Assemblies. J. Phys. Chem. Lett. 2020, 11 (1), 210-216. https://doi.org/10.1021/acs.jpclett.9b03384.

(9) Hill, J. P. Self-Assembled Hexa-Peri-Hexabenzocoronene Graphitic Nanotube. Science 2004, 304 (5676), 1481-1483. https://doi.org/10.1126/science.1097789.

(10) Zhang, W.; Jin, W.; Fukushima, T.; Saeki, A.; Seki, S.; Aida, T. Supramolecular Linear Heterojunction Composed of Graphite-Like Semiconducting Nanotubular Segments. Science 2011, 334 (6054), 340-343. https://doi.org/10.1126/science.1210369.

(11) Singh, V.; Gupta, N.; Hargenrader, G. N.; Askins, E. J.; Valentine, A. J. S.; Kumar, G.; Mara, M. W.; Agarwal, N.; Li, X.; Chen, L. X.; Cordones, A. A.; Glusac, K. D. Photophysics of Graphene Quantum Dot Assemblies with Axially Coordinated Cobaloxime Catalysts. J. Chem. Phys. 2020, 153 (12), 124903. https://doi.org/10.1063/5.0018581.

(12) Yinxiao Yang; Murali, R. Impact of Size Effect on Graphene Nanoribbon Transport. IEEE Electron Device Lett. 2010, 31 (3), 237-239. https://doi.org/10.1109/LED.2009.2039915.

(13) Segawa, Y.; Ito, H.; Itami, K. Structurally Uniform and Atomically Precise Carbon Nanostructures. Nat. Rev. Mater. 2016, 1 (1), 15002. https://doi.org/10.1038/natrevmats.2015.2.

(14) Narita, A.; Feng, X.; Hernandez, Y.; Jensen, S. A.; Bonn, M.; Yang, H.; Verzhbitskiy, I. A.; Casiraghi, C.; Hansen, M. R.; Koch, A. H. R.; Fytas, G.; Ivasenko, O.; Li, B.; Mali, K. S.; Balandina, T.; Mahesh, S.; De Feyter, S.; Müllen, K. Synthesis of Structurally Well-Defined and Liquid-Phase-Processable Graphene Nanoribbons. Nat. Chem. 2014, 6 (2), 126-132. https://doi.org/10.1038/nchem.1819. 
(15) Schwab, M. G.; Narita, A.; Hernandez, Y.; Balandina, T.; Mali, K. S.; De Feyter, S.; Feng, X.; Müllen, K. Structurally Defined Graphene Nanoribbons with High Lateral Extension. J. Am. Chem. Soc. 2012, 134 (44), 18169-18172. https://doi.org/10.1021/ja307697j.

(16) Yang, X.; Dou, X.; Rouhanipour, A.; Zhi, L.; Räder, H. J.; Müllen, K. Two-Dimensional Graphene Nanoribbons. J. Am. Chem. Soc. 2008, 130 (13), 4216-4217. https://doi.org/10.1021/ja710234t.

(17) Vo, T. H.; Shekhirev, M.; Kunkel, D. A.; Morton, M. D.; Berglund, E.; Kong, L.; Wilson, P. M.; Dowben, P. A.; Enders, A.; Sinitskii, A. Large-Scale Solution Synthesis of Narrow Graphene Nanoribbons. Nat. Commun. 2014, 5 (1), 3189. https://doi.org/10.1038/ncomms4189.

(18) Chen, L.; Hernandez, Y.; Feng, X.; Müllen, K. From Nanographene and Graphene Nanoribbons to Graphene Sheets: Chemical Synthesis. Angew. Chem. Int. Ed. 2012, 51 (31), 7640-7654. https://doi.org/10.1002/anie.201201084.

(19) Koga, Y.; Kaneda, T.; Saito, Y.; Murakami, K.; Itami, K. Synthesis of Partially and Fully Fused Polyaromatics by Annulative Chlorophenylene Dimerization. Science 2018, 359 (6374), 435-439. https://doi.org/10.1126/science.aap9801.

(20) Ito, H.; Ozaki, K.; Itami, K. Annulative $\pi$-Extension (APEX): Rapid Access to Fused Arenes, Heteroarenes, and Nanographenes. Angew. Chem. Int. Ed. 2017, 56 (37), 11144-11164. https://doi.org/10.1002/anie.201701058.

(21) Yan, X.; Li, B.; Li, L. Colloidal Graphene Quantum Dots with Well-Defined Structures. Acc. Chem. Res. 2013, 46 (10), 2254-2262. https://doi.org/10.1021/ar300137p.

(22) Diez-Perez, I.; Li, Z.; Hihath, J.; Li, J.; Zhang, C.; Yang, X.; Zang, L.; Dai, Y.; Feng, X.; Muellen, K.; Tao, N. Gate-Controlled Electron Transport in Coronenes as a Bottom-up Approach towards Graphene Transistors. Nat. Commun. 2010, 1 (1), 31. https://doi.org/10.1038/ncomms1029.

(23) Narita, A.; Verzhbitskiy, I. A.; Frederickx, W.; Mali, K. S.; Jensen, S. A.; Hansen, M. R.; Bonn, M.; De Feyter, S.; Casiraghi, C.; Feng, X.; Müllen, K. Bottom-Up Synthesis of Liquid-Phase-Processable Graphene Nanoribbons with Near-Infrared Absorption. ACS Nano 2014, 8 (11), 11622-11630. https://doi.org/10.1021/nn5049014.

(24) Son, Y.-W.; Cohen, M. L.; Louie, S. G. Energy Gaps in Graphene Nanoribbons. Phys. Rev. Lett. 2006, 97 (21), 216803. https://doi.org/10.1103/PhysRevLett.97.216803.

(25) Hu, Y.; Xie, P.; De Corato, M.; Ruini, A.; Zhao, S.; Meggendorfer, F.; Straas $\varnothing$, L. A.; Rondin, L.; Simon, P.; Li, J.; Finley, J. J.; Hansen, M. R.; Lauret, J.-S.; Molinari, E.; Feng, X.; Barth, J. V.; Palma, C.-A.; Prezzi, D.; Müllen, K.; Narita, A. Bandgap Engineering of Graphene Nanoribbons by Control over Structural Distortion. J. Am. Chem. Soc. 2018, 140 (25), 7803-7809.

https://doi.org/10.1021/jacs.8b02209.

(26) Denk, R.; Hohage, M.; Zeppenfeld, P.; Cai, J.; Pignedoli, C. A.; Söde, H.; Fasel, R.; Feng, X.; Müllen, K.; Wang, S.; Prezzi, D.; Ferretti, A.; Ruini, A.; Molinari, E.; Ruffieux, P. Exciton-Dominated Optical Response of Ultra-Narrow Graphene Nanoribbons. Nat. Commun. 2014, 5 (1), 4253. https://doi.org/10.1038/ncomms5253.

(27) Tries, A.; Osella, S.; Zhang, P.; Xu, F.; Ramanan, C.; Kläui, M.; Mai, Y.; Beljonne, D.; Wang, H. I. Experimental Observation of Strong Exciton Effects in Graphene Nanoribbons. Nano Lett. 2020, 20 (5), 2993-3002. https://doi.org/10.1021/acs.nanolett.9b04816.

(28) Jensen, S. A.; Ulbricht, R.; Narita, A.; Feng, X.; Müllen, K.; Hertel, T.; Turchinovich, D.; Bonn, M. Ultrafast Photoconductivity of Graphene Nanoribbons and Carbon Nanotubes. Nano Lett. 2013, 13 (12), 5925-5930. https://doi.org/10.1021/nl402978s.

(29) Ivanov, I.; Hu, Y.; Osella, S.; Beser, U.; Wang, H. I.; Beljonne, D.; Narita, A.; Müllen, K.; Turchinovich, D.; Bonn, M. Role of Edge Engineering in Photoconductivity of Graphene Nanoribbons. J. Am. Chem. Soc. 2017, 139 (23), 7982-7988. https://doi.org/10.1021/jacs.7b03467. 
(30) Chen, Z.; Wang, H. I.; Teyssandier, J.; Mali, K. S.; Dumslaff, T.; Ivanov, I.; Zhang, W.; Ruffieux, P.; Fasel, R.; Räder, H. J.; Turchinovich, D.; De Feyter, S.; Feng, X.; Kläui, M.; Narita, A.; Bonn, M.; Müllen, K. Chemical Vapor Deposition Synthesis and Terahertz Photoconductivity of Low-BandGap N = 9 Armchair Graphene Nanoribbons. J. Am. Chem. Soc. 2017, 139 (10), 3635-3638. https://doi.org/10.1021/jacs.7b00776.

(31) Huang, Y.; Xu, F.; Ganzer, L.; Camargo, F. V. A.; Nagahara, T.; Teyssandier, J.; Van Gorp, H.; Basse, K.; Straas $\varnothing$, L. A.; Nagyte, V.; Casiraghi, C.; Hansen, M. R.; De Feyter, S.; Yan, D.; Müllen, K.; Feng, X.; Cerullo, G.; Mai, Y. Intrinsic Properties of Single Graphene Nanoribbons in Solution: Synthetic and Spectroscopic Studies. J. Am. Chem. Soc. 2018, 140 (33), 10416-10420. https://doi.org/10.1021/jacs.8b06028.

(32) Zhao, S.; Rondin, L.; Delport, G.; Voisin, C.; Beser, U.; Hu, Y.; Feng, X.; Müllen, K.; Narita, A.; Campidelli, S.; Lauret, J. S. Fluorescence from Graphene Nanoribbons of Well-Defined Structure. Carbon 2017, 119, 235-240. https://doi.org/10.1016/j.carbon.2017.04.043.

(33) Zhu, J.; German, R.; Senkovskiy, B. V.; Haberer, D.; Fischer, F. R.; Grüneis, A.; van Loosdrecht, P. H. M. Exciton and Phonon Dynamics in Highly Aligned 7-Atom Wide Armchair Graphene Nanoribbons as Seen by Time-Resolved Spontaneous Raman Scattering. Nanoscale 2018, 10 (37), 17975-17982. https://doi.org/10.1039/C8NR05950K.

(34) Soavi, G.; Dal Conte, S.; Manzoni, C.; Viola, D.; Narita, A.; Hu, Y.; Feng, X.; Hohenester, U.; Molinari, E.; Prezzi, D.; Müllen, K.; Cerullo, G. Exciton-Exciton Annihilation and Biexciton Stimulated Emission in Graphene Nanoribbons. Nat. Commun. 2016, 7 (1), 11010. https://doi.org/10.1038/ncomms11010.

(35) Drummer, M. C.; Singh, V.; Gupta, N.; Gesiorski, J. L.; Weerasooriya, R. B.; Glusac, K. D. Photophysics of Nanographenes: From Polycyclic Aromatic Hydrocarbons to Graphene Nanoribbons. Photosynth. Res. 2021. https://doi.org/10.1007/s11120-021-00838-y.

(36) Zoric, M. R.; Askins, E. J.; Qiao, X.; Glusac, K. D. Strong Electronic Coupling of Graphene Nanoribbons onto Basal Plane of a Glassy Carbon Electrode. ACS Appl. Electron. Mater. 2021, 3 (2), 854-860. https://doi.org/10.1021/acsaelm.0c00978.

(37) Morgenroth, F.; Reuther, E.; Müllen, K. Polyphenylene Dendrimers: From Three-Dimensional to Two-Dimensional Structures. Angew. Chem. Int. Ed. Engl. 1997, 36 (6), 631-634. https://doi.org/10.1002/anie.199706311.

(38) Vo, T. H.; Perera, U. G. E.; Shekhirev, M.; Mehdi Pour, M.; Kunkel, D. A.; Lu, H.; Gruverman, A.; Sutter, E.; Cotlet, M.; Nykypanchuk, D.; Zahl, P.; Enders, A.; Sinitskii, A.; Sutter, P. Nitrogen-Doping Induced Self-Assembly of Graphene Nanoribbon-Based Two-Dimensional and Three-Dimensional Metamaterials. Nano Lett. 2015, 15 (9), 5770-5777. https://doi.org/10.1021/acs.nanolett.5b01723.

(39) Tan, Y.-Z.; Yang, B.; Parvez, K.; Narita, A.; Osella, S.; Beljonne, D.; Feng, X.; Müllen, K. Atomically Precise Edge Chlorination of Nanographenes and Its Application in Graphene Nanoribbons. Nat. Commun. 2013, 4 (1), 2646. https://doi.org/10.1038/ncomms3646.

(40) Bäppler, S. A.; Plasser, F.; Wormit, M.; Dreuw, A. Exciton Analysis of Many-Body Wave Functions: Bridging the Gap between the Quasiparticle and Molecular Orbital Pictures. Phys. Rev. A 2014, 90 (5), 052521. https://doi.org/10.1103/PhysRevA.90.052521.

(41) Wang, S.; Wang, J. Quasiparticle Energies and Optical Excitations in Chevron-Type Graphene Nanoribbon. J. Phys. Chem. C 2012, 116 (18), 10193-10197. https://doi.org/10.1021/jp2125872.

(42) Fauvell, T. J.; Cai, Z.; Kirschner, M. S.; Helweh, W.; Kim, P.; Zheng, T.; Schaller, R. D.; Yu, L.; Chen, L. X. Effects of Intra- and Interchain Interactions on Exciton Dynamics of PTB7 Revealed by Model Oligomers. Molecules 2020, 25 (10), 2441. https://doi.org/10.3390/molecules25102441.

(43) Johnsen, M.; Paterson, M. J.; Arnbjerg, J.; Christiansen, O.; Nielsen, C. B.; Jørgensen, M.; Ogilby, P. R. Effects of Conjugation Length and Resonance Enhancement on Two-Photon Absorption in 
Phenylene-Vinylene Oligomers. Phys Chem Chem Phys 2008, 10 (8), 1177-1191. https://doi.org/10.1039/B715441K.

(44) Bednarz, M.; Reineker, P.; Mena-Osteritz, E.; Bäuerle, P. Theoretical Study of the Size Confinement Effect in Linear $\pi$-Conjugated Oligomers. Chem. Phys. 2007, 342 (1-3), 191-200. https://doi.org/10.1016/j.chemphys.2007.09.052.

(45) Fauvell, T. J.; Zheng, T.; Jackson, N. E.; Ratner, M. A.; Yu, L.; Chen, L. X. Photophysical and Morphological Implications of Single-Strand Conjugated Polymer Folding in Solution. Chem. Mater. 2016, 28 (8), 2814-2822. https://doi.org/10.1021/acs.chemmater.6b00734.

(46) Hogan, C.; Palummo, M.; Gierschner, J.; Rubio, A. Correlation Effects in the Optical Spectra of Porphyrin Oligomer Chains: Exciton Confinement and Length Dependence. J. Chem. Phys. 2013, 138 (2), 024312. https://doi.org/10.1063/1.4773582.

(47) Reed, A. E.; Weinstock, R. B.; Weinhold, F. Natural Population Analysis. J. Chem. Phys. 1985, 83 (2), 735-746. https://doi.org/10.1063/1.449486.

(48) Wan, Y.; Stradomska, A.; Knoester, J.; Huang, L. Direct Imaging of Exciton Transport in Tubular Porphyrin Aggregates by Ultrafast Microscopy. J. Am. Chem. Soc. 2017, 139 (21), 7287-7293. https://doi.org/10.1021/jacs.7b01550.

(49) Settels, V.; Schubert, A.; Tafipolski, M.; Liu, W.; Stehr, V.; Topczak, A. K.; Pflaum, J.; Deibel, C.; Fink, R. F.; Engel, V.; Engels, B. Identification of Ultrafast Relaxation Processes As a Major Reason for Inefficient Exciton Diffusion in Perylene-Based Organic Semiconductors. J. Am. Chem. Soc. 2014, 136 (26), 9327-9337. https://doi.org/10.1021/ja413115h.

(50) Schubert, A.; Settels, V.; Liu, W.; Würthner, F.; Meier, C.; Fink, R. F.; Schindlbeck, S.; Lochbrunner, S.; Engels, B.; Engel, V. Ultrafast Exciton Self-Trapping upon Geometry Deformation in PeryleneBased Molecular Aggregates. J. Phys. Chem. Lett. 2013, 4 (5), 792-796. https://doi.org/10.1021/jz4000752.

(51) Fleming, A. J.; Coleman, J. N.; Dalton, A. B.; Fechtenkötter, A.; Watson, M. D.; Müllen, K.; Byrne, H. J.; Blau, W. J. Optical Spectroscopy of Isolated and Aggregate Hexabenzocoronene Derivatives: A Study of Self-Assembling Molecular Nanowires. J. Phys. Chem. B 2003, 107 (1), 37-43. https://doi.org/10.1021/jp0262618.

(52) Wu, J.; Fechtenkötter, A.; Gauss, J.; Watson, M. D.; Kastler, M.; Fechtenkötter, C.; Wagner, M.; Müllen, K. Controlled Self-Assembly of Hexa- $p$ Eri -Hexabenzocoronenes in Solution. J. Am. Chem. Soc. 2004, 126 (36), 11311-11321. https://doi.org/10.1021/ja047577r.

(53) Hughes, J. M.; Hernandez, Y.; Aherne, D.; Doessel, L.; Müllen, K.; Moreton, B.; White, T. W.; Partridge, C.; Costantini, G.; Shmeliov, A.; Shannon, M.; Nicolosi, V.; Coleman, J. N. High Quality Dispersions of Hexabenzocoronene in Organic Solvents. J. Am. Chem. Soc. 2012, 134 (29), 1216812179. https://doi.org/10.1021/ja303683v.

(54) Reger, D.; Haines, P.; Amsharov, K. Y.; Schmidt, J. A.; Ullrich, T.; Bönisch, S.; Hampel, F.; Görling, A.; Nelson, J.; Jelfs, K. E.; Guldi, D. M.; Jux, N. A Family of Superhelicenes: Easily Tunable, Chiral Nanographenes by Merging Helicity with Planar $\pi$ Systems. Angew. Chem. Int. Ed. 2021, 60 (33), 18073-18081. https://doi.org/10.1002/anie.202103253.

(55) Turro, N. J.; Ramamurthy, V.; Scaiano, J. C. Principles of Molecular Photochemistry: An Introduction; University Science Books: Sausalito, Calif, 2009.

(56) Marian, C. M. Spin-Orbit Coupling and Intersystem Crossing in Molecules: Spin-Orbit Coupling. Wiley Interdiscip. Rev. Comput. Mol. Sci. 2012, 2 (2), 187-203. https://doi.org/10.1002/wcms.83.

(57) Sapir, M.; Donckt, E. V. Intersystem Crossing in the Helicenes. Chem. Phys. Lett. 1975, 36 (1), 108110. https://doi.org/10.1016/0009-2614(75)85698-3.

(58) Schmidt, K.; Brovelli, S.; Coropceanu, V.; Beljonne, D.; Cornil, J.; Bazzini, C.; Caronna, T.; Tubino, R.; Meinardi, F.; Shuai, Z.; Brédas, J.-L. Intersystem Crossing Processes in Nonplanar Aromatic 
Heterocyclic Molecules. J. Phys. Chem. A 2007, 111 (42), 10490-10499. https://doi.org/10.1021/jp075248q.

(59) Mueller, M. L.; Yan, X.; McGuire, J. A.; Li, L. Triplet States and Electronic Relaxation in Photoexcited Graphene Quantum Dots. Nano Lett. 2010, 10 (7), 2679-2682. https://doi.org/10.1021/nl101474d.

(60) Ji, Z.; Dervishi, E.; Doorn, S. K.; Sykora, M. Size-Dependent Electronic Properties of Uniform Ensembles of Strongly Confined Graphene Quantum Dots. J. Phys. Chem. Lett. 2019, 10 (5), 953959. https://doi.org/10.1021/acs.jpclett.9b00119.

(61) Zhu, X.; Su, H. Scaling of Excitons in Graphene Nanoribbons with Armchair Shaped Edges. J. Phys. Chem. A 2011, 115 (43), 11998-12003. https://doi.org/10.1021/jp202787h.

(62) Dimitriev, O. P.; Zirzlmeier, J.; Menon, A.; Slominskii, Y.; Guldi, D. M. Exciton Dynamics in J- and HAggregates of a Tricarbocyanine Near-Infrared Dye. J. Phys. Chem. C 2021, 125 (18), 9855-9865. https://doi.org/10.1021/acs.jpcc.0c11593.

(63) Takeuchi, K. J.; Thompson, M. S.; Pipes, D. W.; Meyer, T. J. Redox and Spectral Properties of Monooxo Polypyridyl Complexes of Ruthenium and Osmium in Aqueous Media. Inorg. Chem. 1984, 23 (13), 1845-1851. https://doi.org/10.1021/ic00181a014.

(64) Elgrishi, N.; Chambers, M. B.; Wang, X.; Fontecave, M. Molecular Polypyridine-Based Metal Complexes as Catalysts for the Reduction of $\mathrm{CO}_{2}$. Chem. Soc. Rev. 2017, 46 (3), 761-796. https://doi.org/10.1039/C5CS00391A.

(65) Mohadjer Beromi, M.; Brudvig, G. W.; Hazari, N.; Lant, H. M. C.; Mercado, B. Q. Synthesis and Reactivity of Paramagnetic Nickel Polypyridyl Complexes Relevant to $\mathrm{C}\left(\mathrm{Sp}^{2}\right)-\mathrm{C}\left(\mathrm{Sp}^{3}\right)$ Coupling Reactions. Angew. Chem. Int. Ed. 2019, 58 (18), 6094-6098. https://doi.org/10.1002/anie.201901866.

(66) Hartley, C. L.; DiRisio, R. J.; Screen, M. E.; Mayer, K. J.; McNamara, W. R. Iron Polypyridyl Complexes for Photocatalytic Hydrogen Generation. Inorg. Chem. 2016, 55 (17), 8865-8870. https://doi.org/10.1021/acs.inorgchem.6b01413.

(67) Chen, Z.; Chen, C.; Weinberg, D. R.; Kang, P.; Concepcion, J. J.; Harrison, D. P.; Brookhart, M. S.; Meyer, T. J. Electrocatalytic Reduction of $\mathrm{CO} 2$ to $\mathrm{CO}$ by Polypyridyl Ruthenium Complexes. Chem. Commun. 2011, 47 (47), 12607. https://doi.org/10.1039/c1cc15071e.

(68) Shimoda, T.; Morishima, T.; Kodama, K.; Hirose, T.; Polyansky, D. E.; Manbeck, G. F.; Muckerman, J. T.; Fujita, E. Photocatalytic $\mathrm{CO}_{2}$ Reduction by Trigonal-Bipyramidal Cobalt(II) Polypyridyl Complexes: The Nature of Cobalt(I) and Cobalt(0) Complexes upon Their Reactions with $\mathrm{CO}_{2}, \mathrm{CO}$, or Proton. Inorg. Chem. 2018, 57 (9), 5486-5498. https://doi.org/10.1021/acs.inorgchem.8b00433.

(69) Shields, B. J.; Kudisch, B.; Scholes, G. D.; Doyle, A. G. Long-Lived Charge-Transfer States of Nickel(II) Aryl Halide Complexes Facilitate Bimolecular Photoinduced Electron Transfer. J. Am. Chem. Soc. 2018, 140 (8), 3035-3039. https://doi.org/10.1021/jacs.7b13281. 
For Table of Contents Only

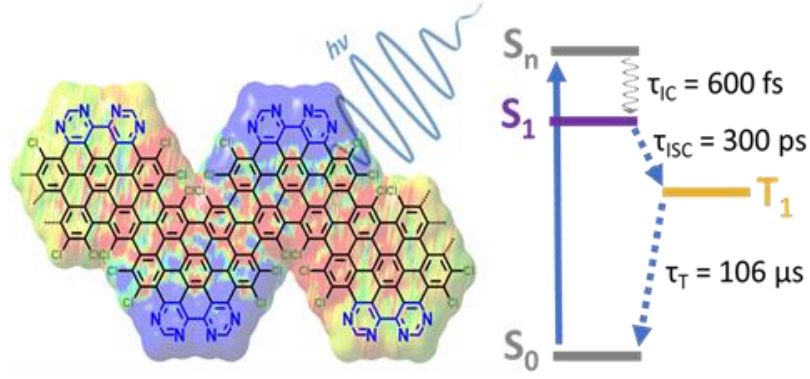

